\title{
Information Theoretic Approaches to Deciphering the Neural Code with Functional Fluorescence Imaging
}

\author{
Jason R. Climer and ${ }^{\circledR D}$ Daniel A. Dombeck \\ https://doi.org/10.1523/ENEURO.0266-21.2021 \\ Department of Neurobiology, Northwestern University, Evanston, 60208 IL
}

\begin{abstract}
Information theoretic metrics have proven useful in quantifying the relationship between behaviorally relevant parameters and neuronal activity with relatively few assumptions. However, these metrics are typically applied to action potential (AP) recordings and were not designed for the slow timescales and variable amplitudes typical of functional fluorescence recordings (e.g., calcium imaging). The lack of research guidelines on how to apply and interpret these metrics with fluorescence traces means the neuroscience community has yet to realize the power of information theoretic metrics. Here, we used computational methods to create mock AP traces with known amounts of information. From these, we generated fluorescence traces and examined the ability of different information metrics to recover the known information values. We provide guidelines for how to use information metrics when applying them to functional fluorescence and demonstrate their appropriate application to GCaMP6f population recordings from mouse hippocampal neurons imaged during virtual navigation.
\end{abstract}

Key words: behavior; calcium imaging; in vivo; information theory; place cells; two-photon microscopy

\section{Significance Statement}

Functional fluorescence imaging and information theoretic quantification could provide a powerful new combination of tools to study neural correlates of behavior, but functional fluorescence signals represent altered versions of the underlying physiological events. Therefore, it is unclear whether or how information metrics can be applied to functional fluorescence imaging data. Here, we performed an in-depth simulation study to examine the application of the widely used bits per second and bits per action potential (AP) metrics of mutual information (MI) to functional florescence recordings. We provide guidelines for how to use information metrics when applying them to functional fluorescence and demonstrate their appropriate application to GCaMP6f population recordings from mouse hippocampal neurons imaged during virtual navigation.

\section{Introduction}

Neurons encode parameters important for animal behavior, at least in part, through the rate of production of action potentials (APs). Evidence for this can be found from electrophysiological AP recordings of orientation tuning in the visual system (Hubel and Wiesel, 2009), chemical sensing in the olfactory system (Leveteau and MacLeod, 1966; Wachowiak and Shipley, 2006), and spatial encoding in the hippocampus (O'Keefe, 1976). Key to

Received June 8, 2021; accepted August 4, 2021; First published August 25, 2021.

The authors declare no competing financial interests. deciphering the neural code, therefore, is defining metrics to quantify the relationship between behavioral parameter spaces and a neuron's spiking rate. There are many metrics used for quantification, and are often used to compare neural responses across conditions or in neurons with complex responses. The underlying assumptions of the different metrics then become important factors to consider when determining which one to use.

Information theory is growing in popularity in the neuroscience community, largely because it provides a means

Author contributions: D.A.D. and J.R.C. designed research; J.R.C. performed research; J.R.C. analyzed data; D.A.D. and J.R.C. wrote the paper. 
to quantify rate coding with relatively few assumptions. One useful information theoretic measure is mutual information (MI), which is typically measured in bits per unit time, and describes the increase in predictability of the neural response when behavioral parameters are known. Formally, $\mathrm{Ml}$ is the information about one variable that can be extracted from another, such as the information about behavior that can be derived from observing neural activity. MI can be applied to neurons with widely varying response properties because it (1) is a nonlinear metric, not requiring the linearity assumptions of correlation metrics (Grubb and Thompson, 2003; Kropff et al., 2015; Hinman et al., 2016); (2) does not assume a response shape, as is typical with Gaussian field mapping metrics (Soo et al., 2011; Kraus et al., 2015; Tang, 2016) or metrics using exponential or polynomial curve fitting (Hinman et al., 2016); and (3) uses the full time trace or shape of the mean response profile, rather than defining receptive fields with thresholding (Niell and Stryker, 2008; Pastalkova et al., 2008; Harvey et al., 2009).

However, Ml can be nontrivial to estimate from neural and behavioral recordings and its estimation is an ongoing area of research (Kraskov et al., 2004; Gao et al., 2017; Belghazi et al., 2018; Timme and Lapish, 2018).

Here, we focus on the most widely used estimator of MI in neuroscience, the SMGM estimator developed by Skaggs, McNaughton, Gothard, and Markus (Skaggs et al., 1993), although as a point of comparison, we also consider the binned estimator (Timme and Lapish, 2018) and a separate technique developed by Kraskov, Stogbauer, and Grassberger (KSG; Kraskov et al., 2004). The binned estimator estimates the joint probability distribution using a 2D histogram of neural response versus behavioral variable; this transforms continuous variables into discrete values (Timme and Lapish, 2018). KSG estimates MI by examining the distance between data-points in the neural activity-behavioral parameter space. The SMGM estimator, on the other hand, relies on the assumption that AP firing follows an inhomogeneous Poisson process. The SMGM estimator therefore requires binning of only the behavioral variable(s), in contrast to the binned estimator. The profile of firing rates versus behavioral variable is then used to estimate the MI.

The relative simplicity of the SMGM estimator has added to its popularity and widespread use in neuroscience applications for estimating behavioral information

This work was supported by The McKnight Foundation, Northwestern University, The Chicago Biomedical Consortium with support from the Searle Funds at The Chicago Community Trust, National Institutes of Health Grants R01MH101297 and T32AG020506, and the National Science Foundation Grant CRCNS1516235.

Acknowledgements: We thank B. Kath and members of the Dombeck Lab for comments on this manuscript and V. Jayaraman, R. Kerr, D. Kim, L. Looger, and K. Svoboda from the GENIE Project (Janelia, Howard Hughes Medical Institute) for GCaMP6.

Correspondence should be addressed to Daniel A. Dombeck at d-dombeck@ northwestern.edu.

https://doi.org/10.1523/ENEURO.0266-21.2021

Copyright (C) 2021 Climer and Dombeck

This is an open-access article distributed under the terms of the Creative Commons Attribution 4.0 International license, which permits unrestricted use, distribution and reproduction in any medium provided that the original work is properly attributed. contained in single unit AP recordings. This metric has proven useful in quantifying rate coding in place cells (Knierim et al., 1995; Markus et al., 1995; Lee et al., 2006; Poucet and Sargolini, 2013), complex spatial responses of hippocampal interneurons (Frank et al., 2001; Wilent and Nitz, 2007), odor sequence cells (Allen et al., 2016), time cells (MacDonald et al., 2013), head direction cells (Stackman and Taube, 1998), speed cells (Fyhn et al., 2002), and face differential neurons (Nguyen et al., 2013, 2014), and has been used across multiple different species (Yartsev and Ulanovsky, 2013; Hazama and Tamura, 2019; Mankin et al., 2019). Furthermore, as a single neuron metric, it provides statistical power for comparisons. Thus, it has been used to quantify differences in rate coding across different brain regions (Simonnet and Brecht, 2019) and across experimental interventions such as lesions (Calton et al., 2003; Liu et al., 2004), inactivations (Huang et al., 2009; Koenig et al., 2011; Brandon et al., 2011; Hok et al., 2013), and applications of drugs (Robbe and Buzsáki, 2009; Newman et al., 2014). Further, it has been used to examine differences in encoding across different behaviors (Zinyuk et al., 2000; Park et al., 2011; Aronov and Tank, 2014) and disease states (Zhou et al., 2007; Gerrard et al., 2008; Fu et al., 2017). SMGM information is often normalized from measuring bits per unit time to instead measure bits per AP. This creates a measure sensitive only to the selectivity of a neuron, and not its average firing rate. Thus, SMGM is a powerful tool for measuring the neural code in electrophysiological recordings of APs.

The power of Ml estimators has yet to be fully exploited by the neuroscience community. For example, the estimators have not yet been widely used to compare encoding properties of large numbers of genetically identified neurons, or to quantify information content of other discrete signaling events such as synaptic inputs; both of which are difficult to study using electrophysiological methods. In vivo imaging of functional indicators has emerged as an important tool, largely because it possesses these capabilities. For example, using fluorescent calcium indicators, the functional properties of large populations of neurons can be simultaneously recorded in rodents (Dombeck et al., 2007; Ziv et al., 2013; Stirman et al., 2016; Sheffield et al., 2017; Radvansky and Dombeck, 2018; Stringer et al., 2019), zebrafish (Ahrens et al., 2013), or invertebrates such as Caenorhabditis elegans (Nguyen et al., 2016) and Drosophila (Keller and Ahrens, 2015; Mann et al., 2017). Furthermore, in vivo imaging can assure the genetic identity of the recorded neurons (Khoshkhoo et al., 2017; Sheffield et al., 2017; Jing et al., 2018a,b,c) and can access subcellular structures, allowing for functional recordings from synapses and dendrites using different functional fluorescent indicators (Sheffield and Dombeck, 2015; Scholl et al., 2017; Sheffield et al., 2017; Jing et al., 2018d; Marvin et al., 2018, 2019; Adoff et al., 2021).

However, these indicators generate signals that are different from the underlying quantal events. For example, somatic calcium indicators reveal intensity variations that are correlated with somatic AP firing rates but are a smoothed and varying amplitude version of the AP train. 
This transformation from AP train to fluorescence trace is an active area of research (Dana et al., 2018; Greenberg et al., 2018; Éltes et al., 2019), but it is often approximated by convolving the AP train with a kernel, which defines the indicator's response to a single AP. The shape of the kernel is a function of the indicator expression level, intracellular calcium buffering, amount of calcium influx, efflux rates, background fluorescence, resting calcium concentration, and other factors. When measured in pyramidal neurons, average kernels typically take the shape of a sharp increase in fluorescence followed by an exponential decay to baseline (Yaksi and Friedrich, 2006; Chen et al., 2013; Park et al., 2013; Dana et al., 2018; Pachitariu et al., 2018). Therefore, while functional fluorescence imaging and information theoretic quantification may prove to be a powerful new combination of tools to study neural correlates of behavior, it is critical to remember that functional fluorescence signals represent altered versions of the underlying physiological events.

Caution is then needed when applying information metrics to continuous functional fluorescence traces, yet the imaging community is already beginning to use information metrics, particularly SMGM. This metric has been applied to somatic calcium responses to compare the information content of the same neurons across different behavioral epochs (Heys and Dombeck, 2018), across different populations of neurons in different brain regions (Hainmueller and Bartos, 2018), across different genetically identified neural populations (Khoshkhoo et al., 2017), or to examine encoding by subcellular structures (Rashid et al., 2020), or to classify the significance of encoding particular parameters by individual neurons (Kinsky et al., 2018; Mau et al., 2018; Rashid et al., 2020).

However, it is essential to recognize some of the assumptions underlying these information metrics are violated by functional florescence recordings. All three metrics (SMGM, KSG, and binned estimation) assume stationarity in the neural response, which is violated by the elongated time responses and relatively slow fluctuations of the fluorescence intensity of the reporters. When applied to spiking data, there is also a change in units: rather than AP counts, functional fluorescence traces are typically plotted in units of florescence change with respect to baseline $(\Delta F / F)$. One possible solution to these issues would be to deconvolve calcium traces to recover APs; however, deconvolution is an active area of research, and the accuracy of these methods has recently been questioned (Evans et al., 2019). Ideally, the calcium traces could be used directly to measure spiking information, without the need for such an in between, potentially error inducing, step.

Quantifying the effects of the above violations on measurements of information using functional fluorescence recordings with an analytical solution is particularly challenging with behaviorally modulated neural recording data. However, a more tractable means of quantifying the effects would be to use a simulation study to measure the induced biases and changes in measurement quality (Morris et al., 2019). This strategy makes use of pseudo-randomly generated AP traces and has the advantage that the ground truth parameters of the simulations are known, while variability because of behavior and other features can be incorporated (Cohen and Kohn, 2011; Climer et al., 2013, 2015; Østergaard et al., 2018).

To provide the field with guidelines for the use of information metrics applied to functional fluorescence recording data, we used computational simulation methods to create a library of ten thousand mock neurons whose spiking output carry an exact, known (ground-truth) amount of information about the animal's spatial location in its environment. We used real behavioral data (available at https:// doi.org/10.7910/DVN/SCQYKR) of spatial position over time from mice navigating in virtual linear tracks and then simulated the spatial firing patterns of the mock neurons using an inhomogeneous Poisson process framework (Brown et al., 2003; Paninski, 2004; Climer et al., 2013). We then simulated fluorescent calcium responses for each neuron in each session by convolving the AP trains with calcium kernels for different indicators, primarily GCamp6f (Chen et al., 2013), and then we added noise. MI metrics (between spatial location and the neural signals) were then applied to the spiking or fluorescence traces to quantify the performance of the metrics for estimating information. We provide a user toolbox (found at https://github.com/ DombeckLab/infoTheory), which consists of MATLAB functions to generate libraries of model neurons with known amounts of information, to generate spiking or fluorescence time-series from those model neurons, and to estimate neuron information from real or model spiking or fluorescence time-series datasets using the three metrics considered here (SMGM, binned estimator, KSG). We focused on testing the performance of the SMGM method, and then compared its performance to the binned estimation and KSG methods, which do not have the underlying Poisson assumption required for the SMGM approach. We also applied a deconvolution algorithm to test its performance. We then applied this analysis to real datasets of hippocampal neuron populations from mice navigating in virtual linear tracks. We quantified the spatial information content of the populations and then performed Bayesian decoding of mouse position from different information containing subsets of this population. Interestingly, we found that the population quantile with the lowest information values were still able to decode mouse position to the closest quarter of the track. Thus, we provide new findings about the neural code for space that were made possible by the information metrics and guidelines that we introduce here.

The SMGM method applied directly to the mean $\Delta F / F$ intensity map appeared to best recover the ground truth information. We provide guidelines for the use of the SMGM metric when applied to functional fluorescence recordings and demonstrate the appropriate application of these guidelines to GCaMP6f population recordings from hippocampal neurons in mice navigating virtual linear tracks.

\section{Materials and Methods}

\section{Toolbox and data availability}

We provide a user toolbox (freely available at https:// github.com/DombeckLab/infoTheory), which consists of 
MATLAB functions to generate libraries of model neurons with known amounts of information, to generate spiking or fluorescence time-series from those model neurons, and to estimate neuron information from real or model spiking or fluorescence time-series datasets using the three metrics considered here (SMGM, binned estimator, KSG). This toolbox also contains tools to generate mock neurons using a binned distribution, avoiding the Poisson assumption of SMGM. Behavioral data used to generate the random traces is freely available at https://doi.org/10.7910/DVN/SCQYKR.

\section{Construction of AP trains with known ground truth information}

To construct mock neurons with ground truth information, we adapted the differential form of the AP information, in bits per AP (Eq. 6). To create a rate map, we first selected an average firing rate and target ground truth information. The mean rate $(\bar{\lambda})$ was always between 0.1 and $30 \mathrm{~Hz}$, the information in bits per AP $\left(I_{A P}^{E}\right)$ between 0 and 6 bits/AP, and the information in bits per second $\left(I_{s}^{E}\right)$ between 0 and 24 . To more evenly sample each of these, we first randomly selected the bits per second $\left(I_{s}^{E}\right)$ or bits per AP $\left(I_{A P}^{E}\right)$ to target. If the information target was in bits per AP, both the information $\left(I_{A P}^{E}\right)$ and mean firing rate $(\bar{\lambda})$ were chosen uniformly. Because the information in bits per second $I_{s}^{E}=\bar{\lambda} l_{A P}^{E}$, the bits per second information was not uniformly sampled in this case. If the target was to be in bits per second, both the bits per AP $\left(I_{A P}^{E}\right)$ and SMGM bits per second $\left(I_{s}^{E}\right)$ measures were first chosen uniformly. Because the rate $\bar{\lambda}=I_{S}^{E} / I_{A P}^{E}$, this was not chosen uniformly. This procedure was repeated to maintain the bounds on $\bar{\lambda}$, resulting in a non-uniform sampling of information. The final distribution (Fig. 1C) was spread acceptably for further analysis.

The rate maps were constructed by spline interpolating across five control points with two anchored at each end of the track, and taking the exponential for each point, and then normalizing by the numerically calculated integral (Fig. 1A,D). To create a map matching the target information, we began with a random spline. The " $y$ " (relative rate) initial position of each node was chosen from a standard normal distribution and the initial " $x$ " (track position) of the three center nodes was chosen uniformly. The nodes were then systematically moved using the MATLAB built in optimizer 'fmincon' with constraints preventing the crossing of the center nodes and keeping them on the track, and the 'OptimalityTolerance' option set to 0 (Fig. 1A). This was accomplished using the 'genExpSpline' function in the toolbox.

We then randomly selected behavioral traces (see below, Behavior) and concatenated sessions until a total time randomly chosen between 3 and 60 min was reached (Figs. 1E, F, 2A, 3A). This was accomplished using the 'loadBehaviorT' function in the toolbox. The track positions were normalized and used to build a conditional intensity function (CIF) from the rate function above. The CIF was normalized to match an expected mean rate over the entire session, and the MATLAB built-in 'poissrnd' function was used to generate AP times, sampled at $1 \mathrm{kHz}$. The was accomplished using the 'genSpikeTrain' function in the toolbox. Finally, the AP times were binned according to the counts within mock imaging frames sampled at $30 \mathrm{~Hz}$.

\section{Simulated $\frac{\Delta F}{F}$ traces}

To construct the $\frac{\Delta F}{F}$ traces (Figs. $1 E, J, K, 2 A, 3 A$ ), we first created a single AP response kernel from the peak-normalized sum of two exponentials:

$$
g(t)=\frac{e^{-a t}-e^{-b t}}{\left(\frac{a}{b}\right)^{\frac{a}{b-a}}-\left(\frac{a}{b}\right)^{\frac{b}{b-a}}},
$$

where $t$ is the time since the AP and $a$ and $b$ are chosen to minimize $\left(1-g\left(\tau_{\text {rise }}\right)\right)^{2}+\left(0.5-g\left(\tau_{\text {rise }}+\tau_{\text {fall }}\right)\right)^{2}$ where $\tau_{\text {rise }}$ is the rise time in seconds and $\tau_{\text {fall }}$ is the half-fall time in seconds. Deviations in $\tau_{\text {rise }}$ and $\tau_{\text {fall }}$ from baseline were also measured. The kernel $\mathrm{g}(\mathrm{t})$ was then multiplied by the indicator height. The kernel parameters were generated using the 'fluorescenceKernel' function, and evaluated using the 'doubleExp' function in the toolbox.

The GCaMP6f, GCaMP6s, and jRGECO1a heights, rise and fall times were measured as responses to single APs in vivo (Kalko et al., 2011; Chen et al., 2013; Dana et al., 2019): other kernels (Fig. 2H; Extended Data Figs. 2-2, 3-1) were approximated from other experiments presented in the references (seen in Table 1).

To define the width of the kernel (Figs. $2 L-N, 3 K-M$ ), we considered the kernel as a low pass filtered version of the APs. If we normalize the filter to mean 1 , it has the Fourier transform $\left(\frac{1}{a+2 \pi f j}-\frac{1}{b+2 \pi f j}\right)\left(\frac{a b}{b-a}\right)$. The kernel width was defined as the $-3-\mathrm{dB}(50 \%)$ cutoff period of this filter: $f^{-1}=\frac{\sqrt{-a^{2}-b^{2}+\sqrt{a^{4}+14 a^{2} b^{2}+b^{4}}}}{2 \pi \sqrt{2}}$. For the simulations with different width kernels, a kernel width was chosen between 0.01 and $10 \mathrm{~s}$, a rise time between 0.001 and $1 \mathrm{~s}$, and a fall time between the rise time and $2 \mathrm{~s}$. Then, $a$ and $b$ were chosen to minimize the squared error between these three targets using the built in MATLAB optimizer 'fminsearch.'

White noise with a SD of $0.15 \frac{\Delta F}{F}$ was then added to the mock fluorescence traces.

\section{Nonlinearity}

In our linear simulations used throughout this work, the fluorescence kernels associated with a fast sequence of APs were approximated to sum linearly. In real cultured neurons, a summation nonlinearity has been observed such that sequences of APs do not generate a linear summation in $\Delta \mathrm{F} / \mathrm{F}$ (Dana et al., 2019). To simulate this nonlinearity, the $\frac{\Delta F}{F}$ trace was then further transformed as: 
A

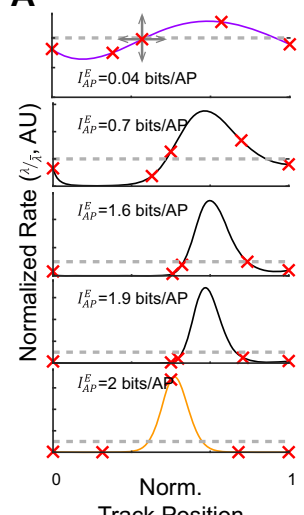

E

1) Randomly select from real behavior library

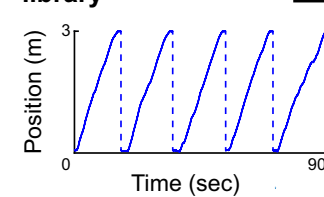

3) Generate AP times as an inhomogenous Poisson process

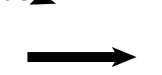

4) Convolve with fit kernel and add noise to create $\Delta \mathrm{F} / \mathrm{F}$ response

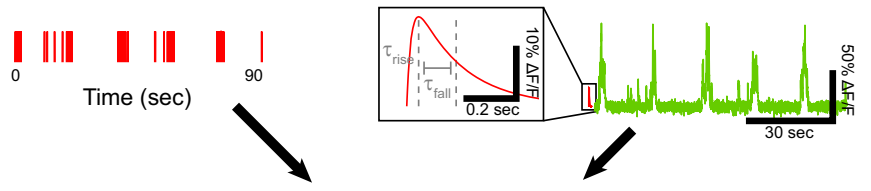

5) Analyze with information theoretic techniques and compare to ground truth and $\Delta F / F$ with spiking
C
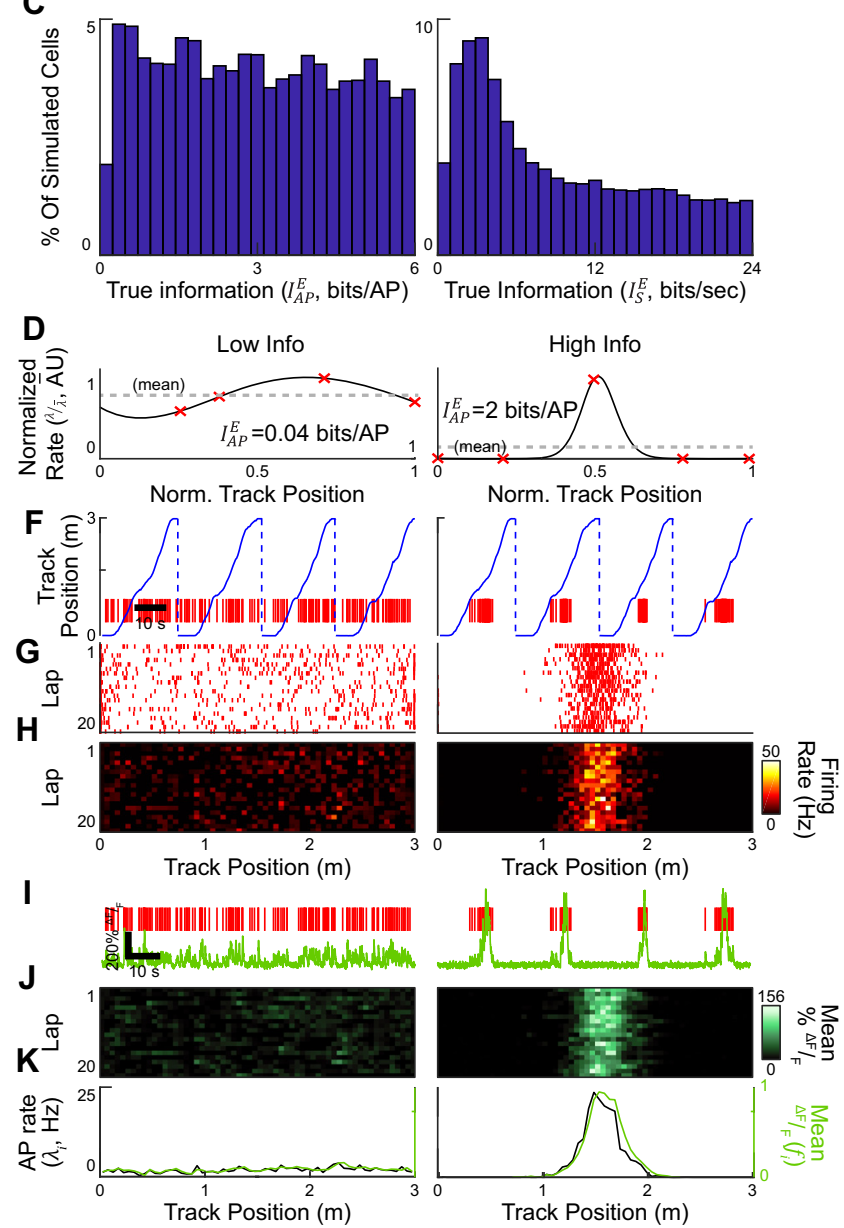

Track Position (m)

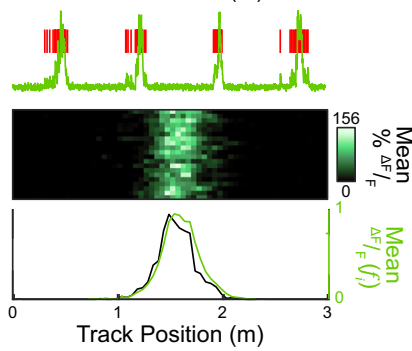

Figure 1. Procedures for generating a library of 10,000 neurons with known amounts of information. $\boldsymbol{A}$, Five splines with a gradient of ground truth information $\left(I_{A P}^{E}\right)$ representing the steps in generating a continuous rate map $(\lambda(x))$ matching the desired target information, in this case, 2 bits/AP. Red Xs indicate control nodes that were moved to change the shape of the spline and minimize the squared error to the target information. $\boldsymbol{B}$, Cross-section of the error surface around the solution point as a function of the position of node 3 , and the trajectory taken by the solver to minimize the error and arrive at the target. $\boldsymbol{C}$, Histograms of ground truth information resulting from repeating the procedure in $\boldsymbol{A}, \boldsymbol{B} 10,000$ times to target a range of ground truth information values in bits per second $\left(I_{S}^{E}\right)$. $\boldsymbol{D}$, Splines representing $\lambda(x)$ ) and bits per AP $\left(I_{A P}^{E}\right.$ at the solution point for a low $\left(I_{A P}^{E}=0.04\right.$ bits/AP, left) and high ( $I_{A P}^{E}, 2$ bits/AP, right) information neuron. $E$, Steps to generate mock AP and functional fluorescence data. (1) An example real behavior trace from a mouse running on a linear track that was used to generate the simulated spiking. (2) The behavior in combination with the rate maps generated in $\boldsymbol{A}-\boldsymbol{D}$ were used to generate an instantaneous firing rate trace. (3) The instantaneous rate was used to pseudorandomly generate APs, as shown in this mock raster. (4) The AP raster was convolved with the GCaMP6f kernel (red, inset), and noise was added to generate a mock $\frac{\Delta F}{F}$ trace. (5) Large numbers of these traces were generated and used to assess the effects of many simulation parameters on the estimators. $\boldsymbol{F}-\boldsymbol{L}$, Spiking and fluorescence activity patterns generated from the example simulated neurons shown in $\boldsymbol{D}$ and using a mean firing rate of $1 \mathrm{~Hz}$. $\boldsymbol{F}$, Behavioral trace in blue with AP raster shown in red. G, Lap-by-lap raster of the neurons' firing versus mouse track position. $\boldsymbol{H}$, Lap by lap binned, firing rates versus mouse track position for the neurons. $\boldsymbol{I}$, AP raster (red) and mock calcium traces for the same behavioral period shown in $\boldsymbol{F}$. $\boldsymbol{J}$, Lap by lap mean binned fluorescence versus mouse position for the neurons. $\boldsymbol{K}$, Binned average firing rate $\left(\lambda_{i}\right.$, black) and fluorescence intensity $\left(f_{i}\right.$, green) maps for the two neurons. These maps were used for information analyses.

$$
\frac{\Delta F^{\prime}}{F}=\operatorname{sign}\left(\frac{\Delta F}{F}\right) * \frac{6.264}{1+e^{-3.251 R e\left(\log _{10}\left(\frac{\Delta F}{F}\right)\right)}} .
$$

This equation was arrived at by fitting the measured responses in Dana et al. (2019; their Fig. 2C), which can be compared with the nonlinearity used here (Extended Data Fig. 3-3A).

\section{Deconvolution}

Deconvolution was performed using the previously described FOOPSI algorithm (Vogelstein et al., 2010; Friedrich et al., 2017). The regularization coefficient was set at 0.02154 , which maximized the correlation between the deconvolved trace and the true spike train in a random sample of 500 simulated traces: all other parameters were 

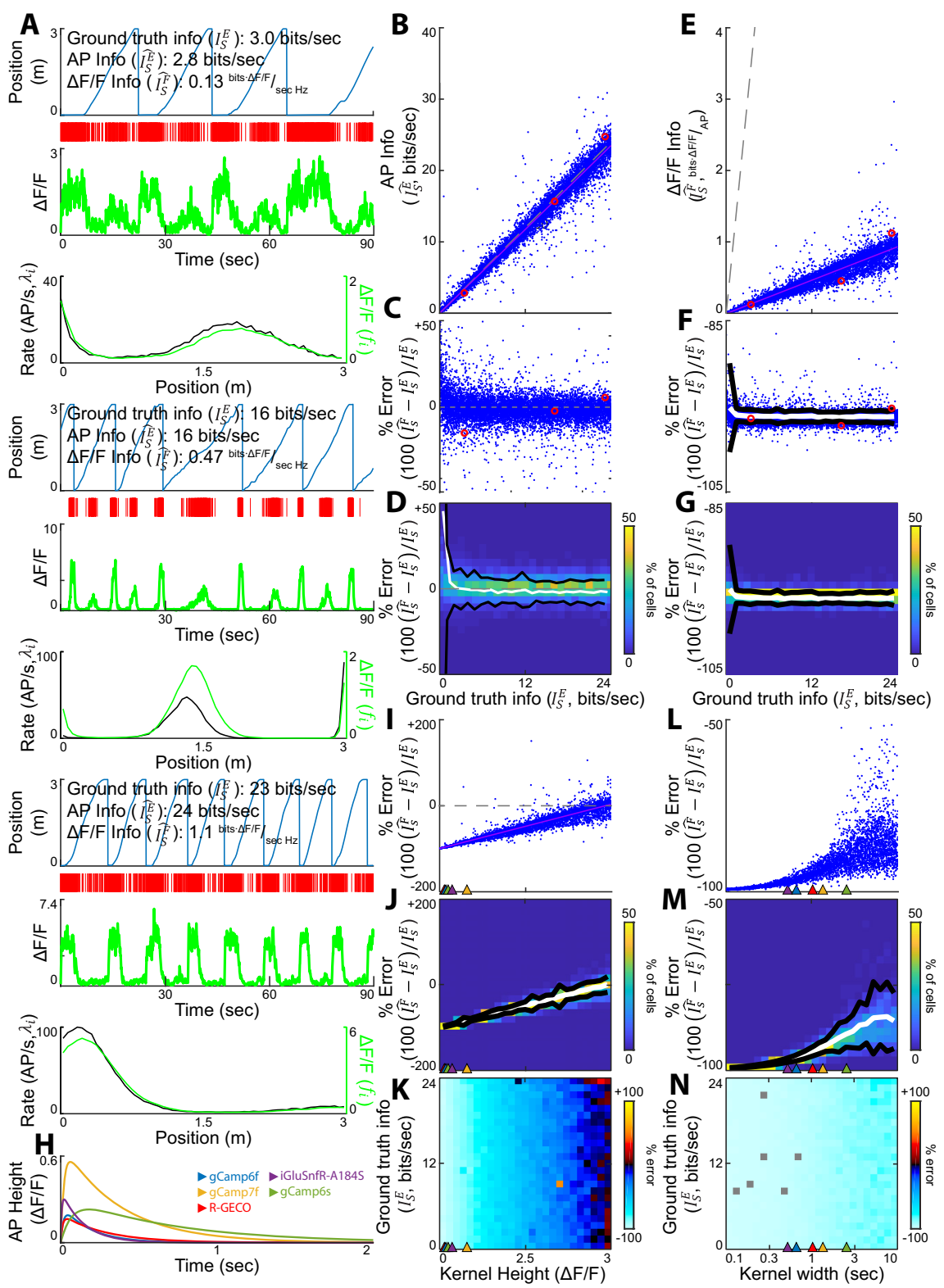

Figure 2. Quantification of the precision of the SMGM bits per second metric using APs or functional fluorescence recordings. $\boldsymbol{A}$, Three representative mock neurons spanning the range of ground truth information values in bits per second $\left(I_{S}^{E}\right)$. From top to bottom for each, Mouse track position versus time, AP raster, fluorescence calcium trace (green), and firing rate map $\left(\lambda_{i}\right.$, black) and change in fluorescence map ( $f_{i}$, green). $\boldsymbol{B}-\boldsymbol{D}$, The ground truth bits per second values are well recovered when measured from AP traces. $\boldsymbol{B}$, Information measured from AP data using the SMGM bits per second metric $\left(I_{S}^{E}\right)$ versus ground truth information $\left(I_{S}^{E}\right)$. Each dot is a single mock neuron, the gray dashed line is the unity line (perfect measurement), the pink line is the line of best fit. Red circles show the examples in $\boldsymbol{A}$. $\boldsymbol{C}$, Percentage error for the information measurements shown in $\boldsymbol{B}$. $\boldsymbol{D}$, Heat map of percentage error measurements shown in $\boldsymbol{C}$. Black lines are 2 SDs, the white line is the mean. $\boldsymbol{E}-\boldsymbol{G}$, Effects of applying the SMGM bits per second metric to fluorescence traces. $\boldsymbol{E}$, Information measured from mock GCaMP6f traces using the SMGM bits per second metric $\left(I_{s}^{F}\right)$ versus ground truth information $\left(I_{s}^{E}\right)$. $\boldsymbol{F}$, Percentage error for the information measurements shown in $\boldsymbol{E}$. G, Heat map of percentage error measurements shown in $\boldsymbol{F}$. $\boldsymbol{H}$, Representative mock kernels mimicking responses from different indicators. $\boldsymbol{I} \boldsymbol{K}$, The effect of kernel height on estimating ground truth information $\left(I_{s}^{E}\right)$ using the SMGM bits per second metric $\left(\hat{I}_{s}^{F}\right)$. Kernel height for the kernels shown in $\boldsymbol{H}$ are indicated by colored triangles. $\boldsymbol{I}$, Percentage error as a function of kernel height. $\boldsymbol{J}$, Heat map of percentage error measurements shown in $\boldsymbol{I}$ with mean (white) and 2 SDs (black). $\boldsymbol{K}$, The average percentage error as a function of kernel height and ground truth information in SMGM bits per second $\left(I_{S}^{E}\right)$. $\mathbf{L}-\boldsymbol{N}$, The effect of kernel width on estimating ground truth information $\left(I_{s}^{E}\right)$ using the SMGM bits per second metric $\left(I_{s}^{F}\right)$. Kernel widths for the kernels shown in $\boldsymbol{H}$ are indicated by colored triangles. $\boldsymbol{L}$, Percentage error as a function of kernel width. $\boldsymbol{M}$, Heat map of percentage error measurements shown in $\boldsymbol{L}$ with mean (white) and 2 SDs (black). $\boldsymbol{N}$, The average percentage error as a function of kernel width. Recording density affected the metrics (Extended Data Figure 2-1). Changing the kernel to common indicators yielded qualitatively similar, but quantitatively different results (Extended Data Figure 2-2). 

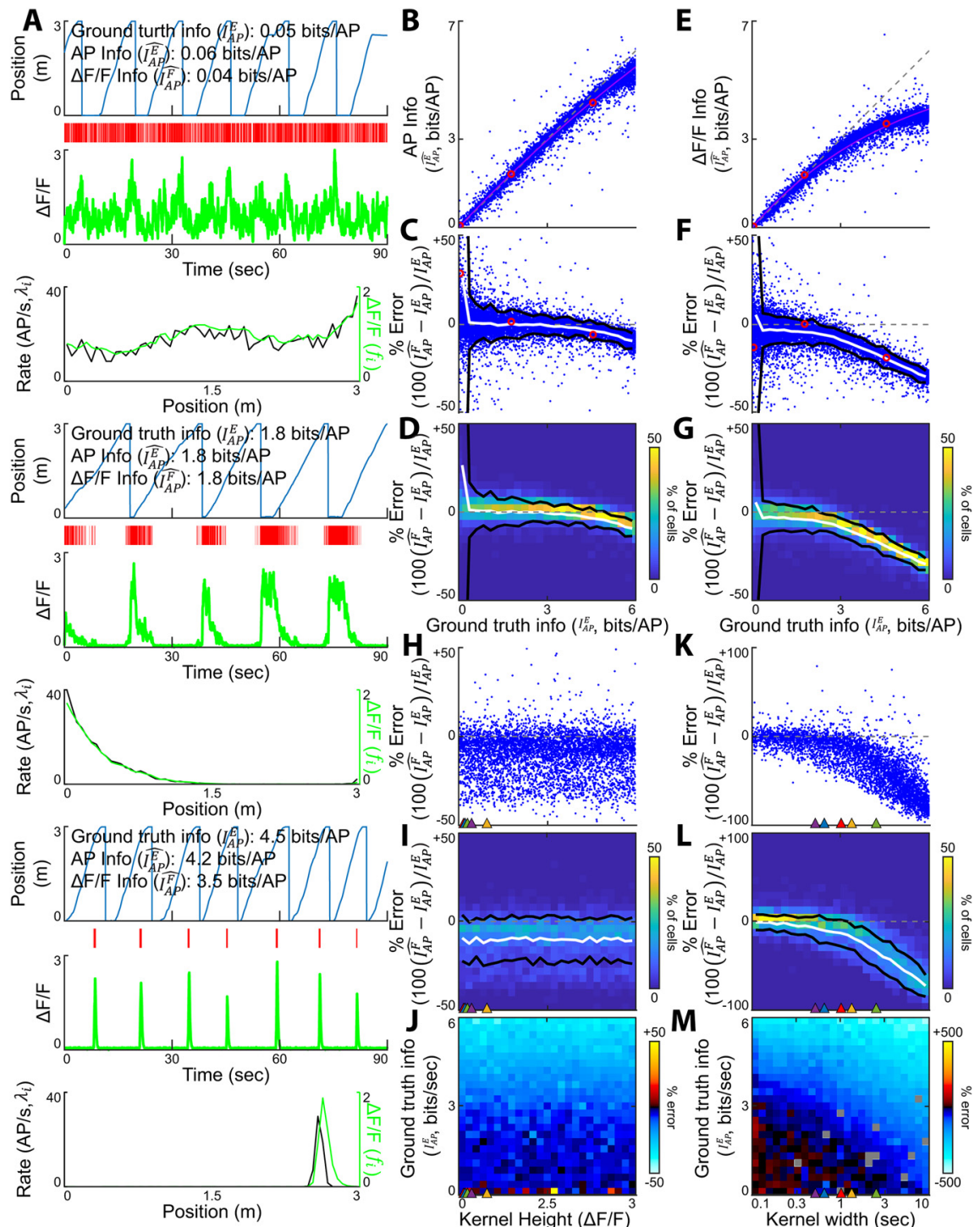

Figure 3. Quantification of the precision of the SMGM bits per AP metric using APs or functional fluorescence recordings. $\boldsymbol{A}$, Three representative mock neurons spanning the range of ground truth information values in bits per AP (I $\left.I_{A P}\right)$. From top to bottom for each, Mouse track position versus time, AP raster, fluorescence calcium trace (green), and firing rate map ( $\lambda_{i}$, black) and change in fluorescence map $\left(f_{i}\right.$, green). $\boldsymbol{B}-\boldsymbol{D}$, The ground truth bits per AP values are well recovered when measured from AP traces. $\boldsymbol{B}$, Information measured from AP data using the SMGM bits per AP metric $\left(I_{A P}^{\hat{E}}\right)$ versus ground truth information $\left(I_{A P}^{E}\right)$. Each dot is a single mock neuron, the gray dashed line is the unity line (perfect measurement). Red circles show the examples in $\boldsymbol{A}$. $\boldsymbol{C}$, Percentage error for the information measurements shown in $\boldsymbol{B}$. $\boldsymbol{D}$, Heat map of percentage error measurements shown in $\boldsymbol{C}$. Black lines are 2 SDs, the white line is the mean. $\boldsymbol{E}-\boldsymbol{G}$, Effects of applying the SMGM bits per AP metric to fluorescence traces. $\boldsymbol{E}$, Information measured from mock GCaMP6f traces using the SMGM bits per AP metric ( $\left.I_{A P}\right)$ versus ground truth information $\left(I_{A P}^{E}\right)$. $\boldsymbol{F}$, Percentage error for the information measurements shown in $\boldsymbol{E}$. $\boldsymbol{G}$, Heat map of percentage error measurements shown in $\boldsymbol{F}$. $\boldsymbol{H}-\boldsymbol{J}$, The effect of kernel height on estimating ground truth information $\left(I_{A P}^{E}\right)$ using the SMGM bits per second metric $\left(I_{A P}\right)$. Kernel height for the kernels shown in Figure $2 H$ are indicated by colored triangles. $\boldsymbol{H}$, Percentage error as a function of kernel height. $\boldsymbol{I}$, Heat map of percentage error measurements shown in $\boldsymbol{H}$ with mean (white) and 2 SDs (black). $\boldsymbol{J}$, The average percentage error as a function of kernel height and ground truth information in bits per AP $\left(I_{A P}^{E}\right) . K-M$, The effect of kernel width on estimating ground truth information $\left(I_{A P}^{E}\right)$ using the SMGM bits per AP metric $\left(I_{A P}\right)$. Kernel widths for the kernels shown in Figure $2 H$ are indicated by colored triangles. $\boldsymbol{L}$, Percentage error as a function of kernel width. $\boldsymbol{M}$, Heat map of percentage error measurements shown in $\boldsymbol{L}$ with mean (white) and 2 SDs (black). $\boldsymbol{N}$, The average percentage error as a function of kernel width. Changing the kernel to common indicators yielded qualitatively similar, but quantitatively different results (Extended Data Figure 3-1). These errors could not be resolved by changing the bin width (Extended Data Figure 3-2). Addition of a nonlinearity further distorted the measured information (Extended Data Figure 3-3). 
Table 1: Properties of indicator kernels used

\begin{tabular}{|c|c|c|c|c|}
\hline & Height & & & \\
\hline Kernel & $\Delta \boldsymbol{F} / \boldsymbol{F}$ & Rise (s) & Fall (s) & Source \\
\hline$\overline{\text { gCaMP6f }}$ & 0.190 & 0.042 & 0.142 & Chen et al. (2013) \\
\hline jRGECO1a & 0.164 & 0.041 & 0.207 & Kalko et al. (2011) \\
\hline gCaMP7f & 0.560 & 0.063 & 0.276 & Dana et al. (2019) \\
\hline gCaMP6s & 0.230 & 0.179 & 0.550 & Chen et al. (2013) \\
\hline iGluSnfR-A184S & 0.300 & 0.022 & 0.106 & Marvin et al. (2018) \\
\hline
\end{tabular}

optimized for each trace. Because the example regularization coefficient provided by Friedrich et al., 2017 was 2.4 , we also measured information values at 100 different values for the regularization coefficient between 0 and 3 ; this had little effect on the measured information (Extended Data Fig. 4-1).

\section{KSG estimator}

The previously described second KSG estimator (Kraskov et al., 2004) was used using the fifth nearest neighbor distance.

\section{Binned estimators}

The binned Ml estimators were used (Timme and Lapish, 2018). The activity trace was divided into 10 bins, either evenly across the span of the activity (uniform binned) or variably so the bins contained the same number of samples (occupancy binned). Position was similarly divided into 60 bins.

\section{Gaussian simulations}

To compare the analytic approximation to our numerical method, the numerical techniques had to be applied to place cells with Gaussian rate maps. The same target information, firing rates, and behavior were used as for our original 10,000 simulations with spline rate maps. However, instead the rate map was chosen as a Gaussian with width $\sigma=e^{\frac{1}{2}\left(-1-\left.2\right|_{A P} ^{E} \log (2)-\log (2 \pi)\right)}$. For the numeric simulations, the true amount of information was calculated using a numeric integrator. The instantaneous rate was calculated using the normal distribution PDF. This was normalized and used to generate a spike train and florescence trace as above.

\section{Bayesian decoding}

The Bayesian decoder used here (Fig. 5G,H) was adapted from a previously described method (Zhang et al., 1998). Decoding was performed on the likelihood that a significant transient occurred in a time frame, trained on the first $80 \%$ of the session and tested on the last $20 \%$. The session was divided into $\Delta t=0.1 \mathrm{~s}$ bins. The conditional likelihood that an animal is in position $x_{i}$ given the number of active frames during a time window $(n)$ is

$$
p\left(x_{i} \mid n\right)=p_{X}\left(x_{i}\right)\left(\prod_{j=1}^{M} f_{i, j}^{n_{j}}\right) e^{-\Delta t} \sum_{j=1}^{M},
$$

Where $p_{X}\left(x_{i}\right)$ is the (marginal) probability that the animal is in the $i^{\text {th }}$ spatial bin during a time sample, $f_{i, j}$ is the average rate of significant frames by the $j^{\text {th }}$ neuron in the $t^{\text {th }}$ spatial bin, $n_{j}$ is the number of significant frames observed during the time window in neuron $j$, and $M$ is the total number of neurons. The decoded position was selected as the one with maximum conditional likelihood.

\section{Animals}

Ten- to 12-week-old male C57BL/6 mice (20-30 g) were individually housed under a reverse $12 / 12 \mathrm{~h}$ light/dark cycle, all experiments were conducted during the dark phase. All experiments were approved by the Northwestern University Animal Care and Use committee.

\section{Behavior}

We used a previously described virtual reality set-up and task (Heys et al., 2014; Sheffield and Dombeck, 2015; Sheffield et al., 2017), some of the behavior sessions used here has previously appeared in these studies. Briefly, water scheduled, head fixed mice were trained to run on a cylindrical treadmill down a 3-m virtual track to receive a water $(4 \mu \mathrm{l})$ reward at the end of the track, and were subsequently teleported to the beginning of the track after a 1.5-s delay. Behavioral sessions were included if the animal ran at least 20 laps containing a continuous $40-\mathrm{cm}$ run for which the velocity was over $7 \mathrm{~cm} / \mathrm{s}$ during a 5- to 30-min session.

\section{Mouse surgery and virus injected}

We performed population calcium imaging of CA1 neurons as described previously (Sheffield and Dombeck, 2015; Sheffield et al., 2017). Briefly, $30 \mathrm{nl}$ of AAV1SynFCaMP6f (University of Pennsylvania Vector Core, 1.5 $\times 10^{13} \mathrm{GC} / \mathrm{ml}$ ) was injected through a small craniotomy over the right hippocampus (1.8 $\mathrm{mm}$ lateral, $2.3 \mathrm{~mm}$ caudal of bregma; $1.25 \mathrm{~mm}$ below the surface of the brain) under isoflurane (1-2\%) anesthesia. $7 d$ later, a hippocampal window and head plate was implanted as described previously (Dombeck et al., 2010).

\section{Two-photon imaging}

Imaging was performed as previously described (Sheffield and Dombeck, 2015; Sheffield et al., 2017). Scanimage four was used for microscope control and acquisition (Pologruto et al., 2003). Time series movies 1024 or $512 \times 256$ pixels) were acquired at $50 \mathrm{~Hz}$. A Digidata1440A (Molecular Devices) with Clampex 10.3 synchronized position on the linear track, reward timing, and the timing of image frames.

\section{Image processing, region of interest (ROI) selection, and calcium transient analysis}

Images were processed as previously described (Sheffield and Dombeck, 2015; Sheffield et al., 2017), with minor modifications. Briefly, rigid motion correction was performed using cross-correlation as in (Dombeck et al., 2010; Miri et al., 2011; Sheffield and Dombeck, 2015), but here using a fast Fourier transform approximation on the full video. ROIs were defined as previously described (Mukamel et al., 2009; $\mu=0.6,150$ principal/independent components, SD 
threshold $=2.5$, SD smoothing width $=1$, area limits $=100$ 1200 pixels). $\frac{\Delta F}{F}$ traces were generated by normalizing around the eighth percentile of a 3-s sliding window. Significant transients from both experimental and mock fluorescence traces were selected by comparing the ratio of amplitudes and durations of positive to negative going transients with a false positive rate $<0.01 \%$ (Dombeck et al., 2010). Mock traces used the histograms generated from the mock gCaMP6f traces (Extended Data Fig. 2-3) or from the specific matching indicator traces (Extended Data Figs. 2-2, 3-1): experimental data histograms were built separately. All subsequent analyses were run using these significant transients.

\section{Behavior analysis}

The mean virtual track velocity was defined as the total virtual track distance covered during the session divided by the total duration of the session; slow and stop periods were included in this metric. All other analyses were restricted to long running periods, where the animal exceeded a virtual track velocity of $4 \mathrm{~cm} / \mathrm{s}$ and ran continuously for at least $40 \mathrm{~cm}$.

\section{Defining place fields}

Place fields were defined by first creating the spatial fluorescence intensity map $\left(f_{i}\right)$ with the $300-\mathrm{cm}$ track divided into $605-\mathrm{cm}$ bins. This map was smoothed via a 3 -bin boxcar. Transients identified during run periods were shuffled in order and to random intervals to create 1000 bootstrapped intensity maps. Candidate fields were defined as regions of the original fluorescence map with values $>99 \%$ of the bootstrapped maps. Fields were then retained if they were between 20 and $120 \mathrm{~cm}$ wide: significant place cells retained at least one field that satisfied these criteria.

\section{Results}

\section{The SMGM information metrics}

Here, we review the derivation of the SMGM information metrics and the underlying assumptions. For illustrative purposes throughout this manuscript, we use the example of spatial encoding in which the firing pattern of neurons carry information about the animal's location along a linear track; however, the derivations, equations and conclusions generalize to encoded variables over other domains and dimensionalities.

Consider a random variable $X$ representing the positions an animal might take, with $x$ being its value measured at one time sample. The positions are subdivided into $N$ spatial bins, such that $x$ can take on the values $\{1,2, \ldots, N\}$. For our analyses, $N=60$. Consider a random variable $Y$ representing the number of APs a neuron might fire, where $y$ is the count measured within a time sample. $y$ can take on the values of $\{0,1, \ldots,+\infty\} . X$ and $Y$ are both discrete. If $X$ and $Y$ both obey the assumption that each time sample is independent (i.e., they are stationary), then the $\mathrm{Ml}$ (I, in bits per sample) between $\mathrm{X}$ and $\mathrm{Y}$ is expressed as follows:

$$
I(X ; Y)=\sum_{i=1}^{N} \sum_{y=0}^{+\infty} p_{X, Y}\left(x_{i}, y\right) \log _{2} \frac{p_{X, Y}\left(x_{i}, y\right)}{p_{X}\left(x_{i}\right) p_{Y}(y)},
$$

where $p_{X}\left(x_{i}\right)$ is the (marginal) probability that the animal is in the $i^{\text {th }}$ spatial bin during a time sample, $p_{Y}(y)$ is the probability that the neuron fires $y$ APs in the time sample, and $p_{X, Y}\left(x_{i}, y\right)$ is the joint probability that the neuron fires $y$ APs and is in the $i^{\text {th }}$ bin. Recall that $p_{X, Y}\left(x_{i}, y\right)=p_{Y \mid X}\left(y \mid x_{i}\right) p_{X}\left(x_{i}\right)$, where $p_{Y \mid X}\left(y \mid x_{i}\right)$ is the conditional probability that the neuron fires $y$ APs given that the animal is in the $i^{\text {th }}$ spatial bin. We can thus rewrite Equation 1 as follows:

$$
I(X ; Y)=\sum_{i=1}^{N} \sum_{y=0}^{+\infty} p_{Y \mid X}\left(y \mid x_{i}\right) p_{X}\left(x_{i}\right) \log _{2} \frac{p_{Y \mid X}\left(y \mid x_{i}\right)}{p_{Y}(y)} .
$$

With the further assumption that the firing of the neuron follows Poisson statistics, we can then estimate the $\mathrm{Ml}$ as follows: let the AP rate (AP/s or $\mathrm{Hz})$ in a single bin be $\lambda_{i}$, and the average across the session be $\bar{\lambda}$. For an arbitrarily small time window $\Delta t$, the probability that an AP occurs in that window is $\operatorname{Pr}(Y=1 \mid x=i)=\lambda_{i} \Delta t$, with the probability that an AP occurs regardless of position as $\operatorname{Pr}(Y=1)=\bar{\lambda} \Delta t$. We can thus rewrite Equation 2 as:

$$
I(X ; Y)=\sum_{i=1}^{N} \lambda_{i} \Delta t p_{X}\left(x_{i}\right) \log _{2} \frac{\lambda_{i}}{\bar{\lambda}} .
$$

By integrating over $1 \mathrm{~s}\left(\int_{0}^{1} I(X ; Y) d \Delta t\right)$, we obtain the first key SMGM metric for spatial information as measured by AP firing, which is in units of bits per second:

$$
\hat{\mathcal{E}_{s}}=\sum_{i=1}^{N} \lambda_{i} p_{x}\left(x_{i}\right) \log _{2} \frac{\lambda_{i}}{\bar{\lambda}}
$$

For notation, we will use a carrot $(\wedge)$ to indicate an information value that is measured from experiment, the superscript ( $E$ in this case) to show the source of the data, and a subscript to show the units/formula used (bits per second in this case). Thus, $I_{s}$ is the information measured via electrophysiology in bits per second. This metric is linearly dependent on the average firing rate of the neuron, and this dependence is often removed through normalization by the average firing rate to obtain the second key metric of spatial information as measured by AP firing, which is in units of bits/(s/Hz), or more commonly, bits per AP:

$$
I_{A P}=\frac{1}{\bar{\lambda}} \sum_{i=1}^{N} \lambda_{i} p_{X}\left(x_{i}\right) \log _{2} \frac{\lambda_{i}}{\bar{\lambda}} .
$$

Therefore, these two key metrics of spatial information are defined completely by quantities that can be experimentally measured: the mean firing rate $(\bar{\lambda})$ from the AP counts over the duration of the recording, the AP firing rate in the $i^{\text {th }}$ bin from the average rate map $\left(\lambda_{i}\right)$, and the 
probability that the animal is in the $i^{\text {th }}$ spatial bin from the normalized occupancy map $\left(p_{x}\left(x_{i}\right)\right)$. The quantity of and noise in these measurements affects the quality of the metric: in particular, undersampling because of low firing rates or low trial counts induces a substantial positive bias (Treves and Panzeri, 1995).

In the derivation of these metrics, there are two key assumptions that are violated by functional fluorescence recordings. First, the recordings do not follow Poisson statistics: instead of discrete counts of APs $(y)$, the functional fluorescence traces consists of a continuous relative change in fluorescence $(\Delta F / F)$, and instead of a firing rate map $\left(\lambda_{i}\right)$ measured in $\mathrm{Hz}$, average intensity maps in units of $\Delta F / F$ are generated. The stationarity assumption is also violated: because of the slow decay, a time sample of the fluorescence traces depend on the previous samples. The violation of these assumptions by functional fluorescence recording will affect the precision and induce biases in the SMGM information metrics. Since these effects have not previously been addressed or quantified, we measured these biases here using a simulation study.

\section{Building a ground truth library of 10,000 neurons with known values of information}

To create a neuron with a known, ground truth information value, it was necessary to generate a continuous (i.e., infinitesimally small bins) rate map $(\lambda(x))$ matching the desired information. To do this, we first normalized the track length to 1 and assumed the animal's occupancy map to be spatially uniform $\left(p_{X}\left(x_{i}\right)=\frac{1}{N}\right)$. We then created an exponentiated cubic spline with five randomly positioned nodes (Fig. 1A) to build a starting continuous map of the normalized instantaneous firing rate, $\lambda \bar{\lambda}(x)$, with the integral normalized to 1 . We calculated the ground truth amount of information in bits per AP as follows:

$$
I_{A P}^{E}=\int_{0}^{1} \frac{\lambda}{\bar{\lambda}}(x) \log _{2}\left(\frac{\lambda}{\bar{\lambda}}(x)\right) d x .
$$

The locations of the five nodes were then systematically varied (see Materials and Methods) to minimize the squared error between the value calculated in Equation 6 and a target amount of information (Fig. $1 A, B$ ), in the end resulting in a mean error of $5.1 \times 10^{-9}$ bits/AP and a mean absolute error of $1.5 \times 10^{-7}$ bits/AP. The rate map at this convergence point was used for further analysis. This procedure was repeated to generate 10,000 mock neurons with a range of (known and ground truth) information values. Note that the value in Equation 5 cannot be higher than when all the APs arrive in one spatial bin; the rate in that bin is $N \bar{\lambda}$. If we assume uniform occupancy $\left(p_{X}\left(x_{i}\right)=\frac{1}{N}\right)$, then the maximum measurable information is $\log _{2} N$, in our case, 5.9 bits/AP with $N=60$ bins. Thus, the information values considered here range between 0 and 6 bits/AP (Fig. 1C). We chose a mean firing rate $(\bar{\lambda})$ for the neurons between 0.1 and $30 \mathrm{~Hz}$, a range observed for a variety of different cortical and hippocampal neurons during behavior (Shafi et al., 2007; DeWeese et al., 2008; O’Connor et al., 2010; Roxin et al., 2011; Buzsáki and Mizuseki, 2014). From Equations 4, 5, the ground truth information in bits per second is $I_{s}^{E}=\bar{\lambda} I_{A P}^{E}$. $I_{s}^{E}$ for these choices resulted in ground truth information values between $I_{s}^{E}=0$ and $I_{s}^{E}=24$ bits per second (Fig. 1C). Example low $\left(I_{A P}^{E}=0.04\right.$ bits $\left./ A P\right)$ and mid $\left(I_{A P}^{E}=2\right.$ bits/AP) rate maps are shown in Figure $1 D$.

These rate maps provided a basis for generating mock AP firing data (and functional fluorescence data, see below). Under real experimental conditions, recording duration and bin sizes are finite and animal occupancy maps $\left(p_{X}\left(x_{i}\right)\right)$ are not spatially uniform. These experimental limitations add error to the estimate of a neuron's ground truth information value. Therefore, to accurately re-create these limitations in our simulation study, we used real behavior datasets from head-restrained mice running along a 3-m virtual linear track for water rewards (acquired as in Sheffield and Dombeck, 2015; Sheffield et al., 2017). Unless otherwise indicated, all values reported will be the mean \pm standard deviation. We selected at random from a library of 574 behavior sessions from mice navigating along familiar tracks and concatenated and truncated these sessions to create behavior sessions uniformly sampled up to $60 \mathrm{~min}$ in duration (average 30.2 \pm 17.1 min), resulting in an average $132 \pm 71.2$ laps per session and an average running speed of $19.3+3.87 \mathrm{~cm} / \mathrm{s}$ (Fig. $1 E 1)$. This behavior, the average firing rate $(\bar{\lambda})$, and the normalized rate map $\left(\frac{\lambda}{\lambda}(x)\right)$ from the mock neurons were used to create an instantaneous firing rate trace (Fig. $1 E 2)$, sampled at $1 \mathrm{kHz}$, from which AP times were generated assuming Poisson firing statistics (Fig. 1E3). An example mock of spiking in response to behavior for low ( 0.04 bits/AP) and mid ( 2 bits/AP) information neurons can be seen in Figure $1 F-H$. From these spiking responses, we then generated mock fluorescence traces by convolving the raster with a double-exponential kernel matching the rise and fall times for GCaMP6f (Chen et al., 2013; Fig. $1 E 4)$ and adding random Gaussian noise to model shot noise. Mock fluorescence traces for the two example neurons in Figure $1 \mathrm{~F}-\mathrm{H}$ can be seen in Figure $1 / \mathrm{J}$. The mock $\mathrm{AP}$ and fluorescence traces were used to create session mean spatial maps, of binned firing rate $\left(\lambda_{i}\right.$ in $\left.\mathrm{Hz}\right)$ and change in fluorescence $\left(f_{i}\right.$ in $\left.\Delta F / F\right)$, for information analyses (Fig. $1 K$ ). By repeating this process, we built a large dataset of spiking and fluorescence traces, generated from our library of mock neurons with known amounts of information and using real animal spatial behavior. With tens of thousands of these mock neuron recordings, we could then assess the effects of many simulation parameters on the information values determined from the metrics including firing rate, session duration, fluorescence kernel shape, and ground truth information value.

\section{Quantification of the accuracy and precision of the SMGM bits per second metric using functional fluorescence recordings}

We first applied the SMGM bits per second metric $\left(\hat{I}_{S}\right)$ to our mock AP recording traces to verify that they can 
recover our ground-truth information values given finite recording durations and bin sizes, and non-uniform animal occupancy maps $\left(p_{X}\left(x_{i}\right)\right)$. Figure $2 A$ shows three mock neurons with ground truth information values of $I_{s}^{E}=3,15$, and 23 bits per second. When the SMGM bits per second metric $\left(I_{s}^{E}\right)$ was applied to the AP traces from these example neurons, the information was well recovered, with $\hat{l}_{s}^{E}=2.8,15$, and 24 bits per second, respectively. The results from these examples also held across the full 10,000 mock neuron library (Figs. $2 B-D$ ), as a linear fit $\left(y\right.$ - intercept $=0.093 \pm 0.040$, intercept $p=4.6 \times 10^{-6}$ bits per second and slope $=0.97 \pm 0.0030$, slope $p \ll 0.01$ ) explained nearly all the variance $\left(R^{2}=0.97\right)$, the average error was $0.22 \pm 1.25$ bits per second $(1.0 \pm 0.69 \%$ error) and the absolute error was $0.64 \pm 1.05$ bits per second $(8.4 \pm 0.69 \%$ error). There is a substantial positive bias for the lowest firing rates and smallest number of trials (Extended Data Fig. 2-1A, $B$ ) which has been previously well characterized (Treves and Panzeri, 1995), with average errors exceeding $+10 \%$ for $<6 \mathrm{~min}$ of recording, mean rate under $0.6 \mathrm{~Hz}$, and under 11 trials. Thus, the SMGM bits per second metric $\left({ }_{\mathrm{I}}\right)$ recovers the ground-truth information well using AP recordings, with the only error coming from finite recording time and variable animal behavior.

We next discuss the changes to the SMGM bits per second metric $\left(I_{s}\right)$ commonly used for application to functional fluorescence traces (Hainmueller and Bartos, 2018; Heys and Dombeck, 2018), and explore the implications of these changes. Most simply, the mean firing rate $(\bar{\lambda})$ and the mean firing rate in a spatial bin $\left(\lambda_{i}\right)$ are replaced by the mean change in fluorescence $(\bar{f})$ and the mean change in fluorescence in a bin $\left(f_{i}\right)$. Making these substitutions in Equation 4 results in the information as measured by functional fluorescence:

$$
\hat{l_{s}}=\sum_{i=1}^{N} f_{i} p_{x}\left(x_{i}\right) \log _{2} \frac{f_{i}}{\bar{f}} .
$$

The fluorescence map $f_{i}$ differs from the firing rate map $\lambda_{i}$ in two ways. First, the fluorescence map is approximated by the firing rate map scaled by a factor $c$, dependent on the height and width of the kernel and measured in units of $\frac{\Delta F / F}{H z}$; and second, it is smoothed by the kernel (Fig. 1E4). If we discount the latter for a moment and focus on the scaling, $f \approx c \lambda$, we can see that substituting $\lambda$ with $c \lambda$ in Equation 4 results in $\hat{l}_{s}=c \hat{l}_{s}$. The units for $\hat{l}_{s}$ are no longer in bits per second, as it has previously been reported (Hainmueller and Bartos, 2018), but are instead in units of $\frac{b^{A t s^{\Delta F}} / F}{\sec H z}$ or $\frac{b_{i t \Delta^{\Delta F}} / F}{A P}$, which are difficult to interpret (see below, Guidelines for application of information metrics to functional fluorescence imaging data). The effect of smoothing is difficult to analytically since it both alters $c$ by changing the average intensity and distorts the firing rate map. Therefore, to fully quantify the impact of convolving an AP recording with a functional fluorescence kernel on recovering ground truth information, we used our mock fluorescence traces.

We applied a GCaMP6f modeled kernel to the 10,000 mock AP traces to generate 10,000 mock fluorescence calcium traces. Figure $2 A$ shows the fluorescence traces generated from three mock neurons with ground truth information values of $I_{s}^{E}=3,15$, and 23 bits per second. The effects of the convolution can be seen in the differences in scaling and shape between the fluorescence maps $f_{i}$ and the firing rate maps $\lambda_{i}$. When the fluorescence metric $\left(\hat{l}_{s}\right)$ was applied to the fluorescence traces from these example neurons, the information recovered was $l_{S}=0.13,0.47$, and $1.1 \frac{\mathrm{bits}^{\Delta F} / F}{A P}$, respectively, indicating significant deviation from the ground truth information values assuming the units are comparable. The results from these examples also held across the full 10,000 mock neuron library (Fig. 2E-G), as there was a clear scaling of the ground truth information and a consistent underestimation with a mean error of $-11.1 \pm 6.7 \mathrm{AU}(-96.0 \pm 1.3 \%$ error). The best-fit line of the measured information $\left(\hat{I}_{s}\right)$ versus the ground truth information $\left(I_{s}^{E}\right)$ had an intercept near $0(0.0029 \pm 0.0016$ $\left.\frac{b_{i t}{ }^{\Delta F} / F}{A P}, p=0.07\right)$. The slope of this fit was $0.039 \pm 12 \mathrm{e}-4$

$\frac{\Delta F T_{F}}{H z}(p \ll 0.01)$, which provides a measure of the scaling factor (c). This error was not corrected for with denser sampling: it remained consistent even at high firing rates and many trials (Extended Data Fig. 2-1C,D). In addition to this scaling effect caused by $c$, smoothing of the rate map could induce nonlinearity in the relationship between and $I_{s}^{E}$. To test for such an effect, we fit the measured information in Figure $2 E$ with a saturating exponential and compared the fits using a likelihood ratio test: the exponential did not significantly improve the fit $\left(\chi_{1}^{2}=0.093, p=0.76\right)$, which indicates that smoothing by the kernel does not induce significant nonlinearities. $c$ is dependent on the height and width (the integral) of the kernel and was measured here as $0.039 \pm 12 \mathrm{e}-4 \frac{\Delta F / F}{\mathrm{~Hz}}$. The consistent, negative bias observed in estimating information with $\hat{l}_{s}^{F}$ (Fig. 2E) would be easy to correct for assuming the $c$ factor, and therefore the kernel, were similar across all measured neurons. This point is considered further below in the Guidelines for application of information metrics to functional fluorescence imaging data. We conclude that ground truth information, as measured by the fluorescence SMGM bits per second metric $\left(\hat{l}_{s}\right)$, is transformed into different units and is linearly scaled by a factor $c$ dependent on the height and width of the kernel.

The amplitude (height) of the change in fluorescence can vary across indicators and conditions. The height of the kernel, given a constant kernel width, should linearly scale $c$ and the error in estimating information with $\hat{l}_{s}$. To explicitly test this prediction, we simulated an additional 5000 fluorescence traces with kernels of varying height (0-3 $\Delta F / F$; Fig. $2 I-K)$, but that maintain the same shape and width (from the GCaMP6f kernel), and then measured the percent error in estimating information with $\hat{I}_{s}$. As observed above for the GCaMP6f example (Fig. $2 E-G$ ), the percent error in estimating information with $\hat{F}_{s}^{F}$ shows little dependence on ground truth information (Fig. 2I-K). 
However, as a function of the height of the kernel, the percent error (averaged over all ground truth information values) in estimating information with $\hat{l}_{s}$ is fit well with an increasing linear function (intercept $=-99.8 \pm 0.42 \%$, intercept $p \ll 0.01$, slope $20.7 \pm 0.14 \% / \frac{\Delta F}{F}$, slope $p \ll 0.01$, $R^{2}=0.80$; Fig. $\left.2 /, J\right)$. Over the wide array of available functional fluorescent indicators in use today (Fig. $2 H$ ), this leads to differences in error because of differences in transient height of the indicator used alone. For the indicators shown in Figure $2 \mathrm{H}$, there is an average height of $0.603 \pm 0.10 \mathrm{SD} \Delta F / F$ : the error spans from $-95.8 \%$ for the kernel height reported for gCamp6f $(0.19 \Delta F / F)$ to $-88.2 \%$ for gCamp7f $(0.56 \Delta F / F)$. It should be noted that fluorescence $(\Delta F / F)$ is always reported here as a fractional change, not as a percentage (\% $\Delta F / F)$; if a kernel height of $19 \% \Delta F / F$ is used, the units would again change. Thus, as expected, the percent error in estimating information with the SMGM bits per second estimator $\left(\hat{I}_{S}\right)$ scales linearly with the height of the kernel.

The width of the kernel can vary widely across fluorescent indicators (Fig. $2 \mathrm{H}$ ), with "faster" indicators boasting shorter rise and fall times. The combined effect of a longer rise and fall time is to smooth and delay the AP train; in other words, it acts as a causal low-pass filter. The cutoff period of this low pass filter provides a measurement of the effective width of the kernel (see Materials and Methods). The effect of such differences in kernel shape on the error in estimating information with $\hat{l}_{s}$ is difficult to measure analytically. We therefore simulated an additional 5000 fluorescence traces with kernels of different kernel widths (but constant height of the GCaMP6f kernel), resulting in a range of kernel durations (rise times: $1 \mathrm{~ms}$ to $1 \mathrm{~s}$, fall times longer than the rise time up to $2 \mathrm{~s}$ ), and then we measured the percent error in estimating information with $\hat{l}_{s}$. Similarly, as observed above for the GCaMP6f and varying kernel height examples (Fig. 2E-K), the percent error in estimating information with $\hat{l}_{s}$ shows little dependence on ground truth information (Fig. 2N). Interestingly, the percent error (averaged over all ground truth information values) in estimating information with $\hat{l}_{s}$ shows a complex nonlinear response as a function of the width of the kernel (Fig. $2 L, M$ ). The error increases up to a kernel width of $\sim 3 \mathrm{~s}$, at which point it saturates at approximately $-85 \%$ error. This arises from an interaction between changing the average value of the original AP trace and flattening the average fluorescence map $\left(f_{i}\right)$. Over the wide array of available functional fluorescent indicators in use today, this leads to differences in error because of differences in width of the indicator used alone. For example, an average error of $-97.1 \pm 0.63 \%$ were observed for iGluSnfR, the shortest indicator considered here at $0.52 \mathrm{~s}$. For gCamp6s, the slowest indicator examined $(2.54 \mathrm{~s})$, the average was $-89.6 \pm 4.6 \%$. To estimate the percent errors for these five indicators considering differences in both height and duration, we used these five kernels to generate mock fluorescence traces from the 10,000 neurons in Figure $2 B-G$. The resulting distributions, estimated $c$ values, and mean and absolute errors can be seen in Extended Data Figure 2-2. In summary, we conclude that information, as measured by the fluorescence SMGM bits per second metric $\left(\hat{I}_{S}\right)$, is transformed into different units and is linearly scaled by a factor $(c)$ dependent on the height and width of the kernel, with $c$ linearly dependent on height and nonlinearly dependent on width. The error induced by these transformations changes substantially over the range of kernel values of the different functional indicators widely used today, and therefore these are important factors to consider when designing and interpreting functional imaging experiments (for further discussion, see below, Guidelines for application of information metrics to functional fluorescence imaging data).

\section{Quantification of the accuracy and precision of the SMGM bits per AP metric using functional fluorescence recordings}

The SMGM metric is commonly normalized by the mean rate to obtain a measurement in units of bits per AP. We thus applied the SMGM bits per AP metric $\left(I_{A P}\right)$ to our mock AP recording traces to verify that they can recover our ground-truth information values. Figure $3 A$ shows three mock neurons with ground truth information values $I_{A P}^{E}=0.05,1.8$, and 4.2 bits/AP. When the SMGM bits per AP metric $\left(I_{A P}^{\hat{E}_{P}}\right)$ was applied to the AP traces from these example neurons, the information was well recovered, with $I_{A P}^{\hat{E}_{P}}=0.06,1.8$, and 4.2 bits/AP, respectively. The results from these examples also held across the full 10,000 mock neuron library (Fig. $3 B-D$ ), as a linear fit (y - intercept $=0.087 \pm 0.029$, intercept $p=2.8 \mathrm{e}-184$ bits per second and slope $=0.93 \pm 0.0010$, slope $p \ll 0.01$ ) explained nearly all the variance $\left(R^{2}=0.99\right)$, the average error was $-0.071 \pm 0.23$ bits/AP $(3.2 \pm 5.9 \%$ error $)$ and the absolute error was $0.13 \pm 0.21$ bits per second (8.1 $\pm 9 \%$ error). However, the data were better fit with a saturating exponential $\left(\chi_{1}^{2}=1.6 \mathrm{e} 3, p \ll 0.01\right)$ converging to $5.8 \mathrm{bits} / \mathrm{AP}$ as it approached the limit because of the finite bin count. There is a substantial positive bias for the lowest firing rates and smallest number of trials (Extended Data Fig. 2-1E,F) which has been previously well characterized (Treves and Panzeri, 1995). Thus, the SMGM bits per-AP metric $\left(I_{A P}\right)$ recovers the ground-truth information well using AP recordings (except at the largest ground truth information values), with the primary error coming from finite recording time and variable animal behavior.

We next discuss the changes needed to apply the SMGM bits per AP metric $\left.\left(I_{A P}\right)^{\prime}\right)$ to functional fluorescence traces and explore the implications of these changes. Most simply, the mean firing rate $(\bar{\lambda})$ and the mean firing rate in a spatial bin $\left(\lambda_{i}\right)$ are replaced by the mean change in fluorescence $(\bar{f})$ and the mean change in fluorescence in a bin $\left(f_{i}\right)$. Making these substitutions in Equation 5 results in the information as measured by functional fluorescence: 


$$
I_{A P}=\frac{1}{\bar{f}} \sum_{i=1}^{N} f_{i} p_{X}\left(x_{i}\right) \log _{2} f_{i} .
$$

As discussed above, the fluorescence map $\left(f_{i}\right)$ can be approximated as a scaled version of the rate, that is, $f=c \lambda$ and $\bar{f}=c \bar{\lambda}$. Thus, under this approximation, the $c$ factors in Equation 8 cancel, leading to $I_{A P}$ equivalent to $I_{A P}^{\hat{E}}$, with the same units of bits/AP. This, of course, ignores the fact that the kernel smooths the rate map, leading to a bias in the metric that is difficult to quantify analytically.

We then applied the fluorescence SMGM bits per AP metric $\left(I_{A P}\right)$ to our 10,000 mock GCaMP6f traces. Figure $3 A$ shows the fluorescence traces generated from three mock neurons with ground truth information of $I_{A P}^{E}=0.05$, 1.8 , and 4.2 bits/AP. When the fluorescence metric $\left(l_{A P}\right)$ was applied to the fluorescence traces in these examples, the information recovered was $0.04,1.8$, and 3.5 bits/AP, indicating some deviations, especially for the highest information neuron. These results held for the 10,000 mock neuron library (Fig. 3E-G). At low information values, there was little bias, but at higher information values the information recovered was substantially lower than the ground truth information. The mean resulting error was $-0.38 \pm$ 0.58 bits/AP $(-9.7 \pm 27.8 \%)$ and absolute error of 0.39 $(12.9 \pm 26.4 \%)$. This error was better fit with a saturating exponential than a linear fit $\left(\chi_{1}^{2}=1.6 \mathrm{e} 3, p \ll 0.01\right)$, with the average error $<5 \%$ up to ground truth information of 1.8 bits/AP and $<10 \%$ up to 3.0 bits/AP. At ground truth information values higher than 3 bits/AP, the average error was $-1.06 \pm 0.595(-22.5 \pm 9.44 \%)$ and absolute error was $1.07 \pm 0.589$ bits/AP $(22.6 \pm 9.21 \%)$. This error persisted even with denser sampling: it remained consistent even at high firing rates and many trials (Extended Data Fig. 2-1E,F). Thus, the indicator induces relatively little error at lower information values $(<3$ bits/AP), but the smoothing effect of the kernel induces a nonlinear, negative bias to the estimator, particularly at ground truth information values over 3 bits/AP.

Although the height of the kernel can vary between different functional fluorescence indicators (Fig. 2H), these height variations linearly scale the fluorescence map.

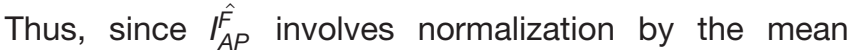
change in fluorescence $(\bar{f}), I_{A P}$ should not depend on kernel height. To explicitly test this prediction, we used the 5000 fluorescence traces described in the previous section (Quantification of the accuracy and precision of the SMGM bits per second metric using functional fluorescence recordings), with kernels of varying height (0-3 $\Delta \mathrm{F} /$ $\mathrm{F}$ ), but that maintain the same shape and width (from the GCaMP6f kernel). Then, we measured the percent error in estimating information with $I_{A P}^{F}$ (Fig. $\left.3 H-J\right)$. Unlike for the SMGM bits per second metric, the percent error (averaged over all ground truth information values) in estimating information with $I_{A P}$ shows little or no dependence on the height of the kernel $(p=0.43)$, but a nonlinear dependence on ground truth information as in Figure $3 E-G$, with no significant difference in the parameters of the saturating exponential fit $\left(\chi_{2}^{2}=1.67, p=0.43\right)$. Thus, as expected, the percent error in estimating information with the SMGM bits per AP metric $\left(I_{A P}\right)$ does not vary with the height of the kernel.

With little effect of kernel height on $I_{A P}$, the width of the kernel likely drives biases in the metric. We thus used the 5000 fluorescence traces generated from a range of different kernel durations (rise times: $1 \mathrm{~ms}$ to $1 \mathrm{~s}$, fall times longer than the rise time up to $2 \mathrm{~s}$, but constant height of the GCaMP6f kernel) from the previous section (Quantification of the accuracy and precision of the SMGM bits per second metric using functional fluorescence recordings), and then we measured the percent error in estimating information with $l_{A P}^{\hat{F}_{p}}$ (Fig. $3 K-M$ ). Similarly, as observed above for GCaMP6f and the varying kernel height examples (Fig. $3 E-J$ ), the percent error in estimating information with $\hat{l}_{s}$ shows a nonlinear dependence on ground truth information (Fig. 3E-G). The percent error (averaged over all ground truth information values) showed a nonlinear response as a function of the width of the kernel (Fig. $3 K, L)$, with a steep increase in error for kernel widths $>\sim 1 \mathrm{~s}$. Even for kernel widths $<\sim 1 \mathrm{~s}$, the percent error was strongly dependent on the ground truth information value, with steep increases in error for values more than $\sim 2.5-3$ bits/AP (Fig. 3M). Thus, as the kernels gets wider, there is more negative bias at lower and lower information measured. The resulting errors are thus larger for wider kernel indicators, for example, with a kernel width the same as gCaMP6s (2.54s), the error exceeds $-17 \%$ even at low $(<0.25$ bits/AP) information, with average errors of $-0.86 \pm 1.0$ bits/AP $(-31 \pm 19 \%$ error) and absolute errors of $0.87 \pm 1.0$ bits/AP (32.6 $\pm 16 \%$ error). In contrast, with a kernel width the same as iGluSnfR $(0.52 \mathrm{~s})$, the average error exceeded $5 \%$ at 3 bits/AP and $10 \%$ at 3.7 bits/AP with a mean error of $-0.57 \pm 1.00$ $(-8.0 \pm 14 \%)$ bits/AP and absolute error of $0.41 \pm$ 0.56 bits/AP $(11 \pm 11 \%)$. To estimate the percent errors for the five indicators shown in Figure $2 \mathrm{H}$, taking into account differences in both height and duration, we used the five kernels to generate mock fluorescence traces from the $10 \mathrm{k}$ neurons in Figure $3 B-G$. The resulting distributions, mean and absolute errors, and error thresholds can be seen in Extended Data Figure 3-1.

Since the known information values in our library of 10,000 mock neurons were determined using the SMGM metric, which includes the assumption that neuron firing follows an inhomogeneous Poisson process, we next investigated whether the biases observed between AP and fluorescence metrics $\left(I_{S}^{E}\right.$ vs $I_{S}^{F}$ and $I_{A P}$ vs $I_{A P} \hat{F}_{F}$ Figs. 2, 3) in our mock neuron datasets were also observed in real neuron recordings (i.e., real spiking that could deviate from Poisson firing). We therefore measured information in a real spiking dataset from hippocampal neurons in rats running on a behavioral track (Chen et al., 2016; Grosmark and Buzsáki, 2016; Grossmark et al., 2016). We generated mock fluorescence traces as we did with simulated AP trains from our mock neurons, compared the information measured from APs versus fluorescence $\left(\hat{I}_{S}^{E}\right.$ vs 
$I_{S}^{F}$ and $I_{A P}$ vs $\left.I_{A P}\right)$ in the real neuron recordings and found that the biases were largely consistent with the simulated mock neuron datasets (Extended Data Fig. 5-1).

In summary, we conclude that ground truth information, as measured by the fluorescence SMGM bits per AP metric $\left(\hat{F}_{A P}\right)$, retains the units and insensitivity to height scaling of the electrophysiological metric $\left(\hat{I}_{A P}\right)$, but is nonlinearly biased by the smoothing of the fluorescence map dictated by the width of the kernel. The estimation errors strongly depended on both the width of the kernel and the information value being measured. Since these parameters change substantially over the different functional indicators and different neuron types and behaviors that are commonly used today, they are important factors to consider when designing and interpreting functional imaging experiments (see below for further discussion).

\section{Nonlinearity introduces further biases}

The results presented in the previous two sections rely on the approximation that $\Delta F / F$ scales linearly with the firing rate, which is not strictly true in practice (Dana et al., 2018; Greenberg et al., 2018; Éltes et al., 2019). Calcium imaging can be more responsive to bursts of APs rather than isolated spikes, and saturates at high firing rates. As an example for how to examine how nonlinearities between $\Delta F / F$ and firing rate could affect the fluorescence SMGM metrics $\left(\hat{I}_{S}\right.$ and $\left.I_{A P}\right)$, we applied a log-sigmoid nonlinearity (Extended Data Fig. 3-3A) to the 10,000 mock GCaMP6f time-series traces described above, based on the real behavior of GCaMP6f in cultured neurons (Dana et al., 2019; see Materials and Methods). While the resulting measurements (Extended Data Fig. 3-3) of ground truth information, as measured by the fluorescence SMGM metrics, are largely consistent with the results observed when using the linear assumption (Figs. 2, 3), some quantitative difference can be seen. Thus, even a relatively simple nonlinearity between $\Delta F / F$ and firing rate can add distortions to the amount of information measured using the fluorescence SMGM approach.

\section{Deconvolution may not be sufficient to eliminate biases}

The framework presented here for comparing ground truth information with information measured with the SMGM metrics can be extended to test the efficacy of other strategies for extracting MI. In particular, a perfect AP inference method would alleviate the problems associated with applying the SMGM metrics to functional fluorescence recordings. To test the utility of such a strategy in measuring information, we applied a popular deconvolution algorithm, FOOPSI (Vogelstein et al., 2010; see Materials and Methods), to the same 10,000 mock GCaMP6f time-series traces described above. Importantly, this deconvolution algorithm (and other available algorithms) does not recover traces of relative spike probability or exact spikes times, but instead produces sparse traces with arbitrary units, that have non-zero values estimating the relative "intensity" of spike production over time $(d)$. This signal can be thought of as a scaled estimate of the number of spikes per time bin, and thus the average intensity map will have some similar properties to the florescence intensity maps, that is, we would expect the intensity maps from deconvolution to approximate the relative firing rate scaled by some factor $c$, which has arbitrary units.

We then measured information in these deconvolved $d$-traces using the SMGM metrics $\left(l_{s}^{d}\right.$ and $\left.l_{A P}^{\hat{d}}\right)$, which are identical to $\left(\hat{I}_{S}^{F}\right.$ and $\left.I_{A P}\right)$, except SMGM is applied to $d$-traces instead of functional fluorescence traces. When using the SMGM bits per second measure $\left(l_{s}^{d} ;\right.$ Fig. $\left.4 A\right)$, we found a clear scaling of the ground truth information. The scaling factor was very small $(c=1.15 \mathrm{e}-3 \pm 1.8 \mathrm{e}-5 \mathrm{AU})$, resulting in low predicted information (mean error $-11.4 \pm 6.92 \mathrm{AU}$, mean \% error $-99.8 \pm 0.38 \%)$. This error was larger than when we measured information directly from the florescence traces using $\hat{I}_{s}^{F}$ (Fig. 2E; 96.0\% absolute error, rank-sum $p \ll 0.01 ; c=0.0390$ ). It is worth noting that the deconvolved trace $d$ can be arbitrarily scaled, so in a sense this error is arbitrary. However, these are the results from the scaling chosen by a widely used deconvolution algorithm and the large error emphasize that the scale of $d$ can have a large effect on the bits per second measure $\left(l_{s}^{\hat{d}}\right)$.

Assuming that the intensity map of the deconvolved $d$-traces are a scaled version of the true rate maps, we could measure information using the SMGM bits per AP metric $l_{A P}^{\hat{d}}$ without changing units (Fig. 4B). Compared with the SMGM bits per AP metric applied to florescence $\left(I_{A P}^{F}\right)$, on average there was some reduction in the nonlinearity at higher ground truth information $\left(I_{A P}^{E}\right)$ values when using $l_{A P}$, resulting in linear fits closer to the unitary line $\left(I_{A P}^{\hat{d}}\right.$ slope $=1.02 \pm 0.0027, R^{2}=0.76$ vs $I_{A P}$ slope $\left.=0.78 \pm 0.0011, R^{2}=0.93\right)$. However, information measured with $l_{A P}^{\hat{d}}$ was still better fit with a saturating exponential $\left(\chi_{1}^{2}=2.3 \mathrm{e}, p \sim 0\right)$ converging to a saturation value of 5.51 bits/AP (compared with 5.78 for $\left(\hat{F}_{A P}\right)$, as expected since the algorithm is not expected to resolve spikes at orders of magnitude shorter timescales than the kernel. This resulted in a positive bias at lower levels of ground truth information. For ground truth information values below 3 bits/AP, the average error for $l_{A P}^{\hat{d}}$ was $0.556 \pm 0.50 \mathrm{bits} /$ AP $(68.9 \pm 104 \%)$ compared with $-0.060 \pm 0.13$ bits/ $\operatorname{AP}(2.75 \pm 0.36 \%)$ for $l_{A P}$. For ground truth information values above 3 bits/AP, the average error for $l_{A P}^{\hat{d}}$ was $-0.127 \pm 0.76$ bits/AP $(-1.0 \pm 16.8 \%)$ as compared with $-1.04 \pm 0.59$ bits/AP $(-0.22 \pm 9.4 \%)$ for $I_{A P}^{\hat{F}}$. Overall, there was more error when the SMGM bits/AP metric was applied to deconvolved data compared with when applied directly to fluorescence traces $\left[I_{A P}^{\hat{d}}\right.$ mean absolute error $0.60 \pm 0.47 \mathrm{bits} / \mathrm{AP}(52.7 \pm 89.1 \%)$ versus $I_{A P}$ was $0.13 \pm 0.21$ bits per second $(8.1 \pm 9 \%$ error), rank-sum $p \ll 0.01]$. Thus, when comparing the recovery of ground truth information from functional fluorescence traces using 
FOOPSI+SMGM (bits/sec)
FOOPSI+SMGM (bits/AP)
KSG

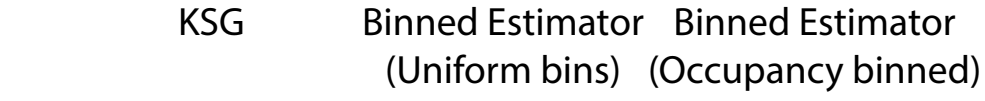

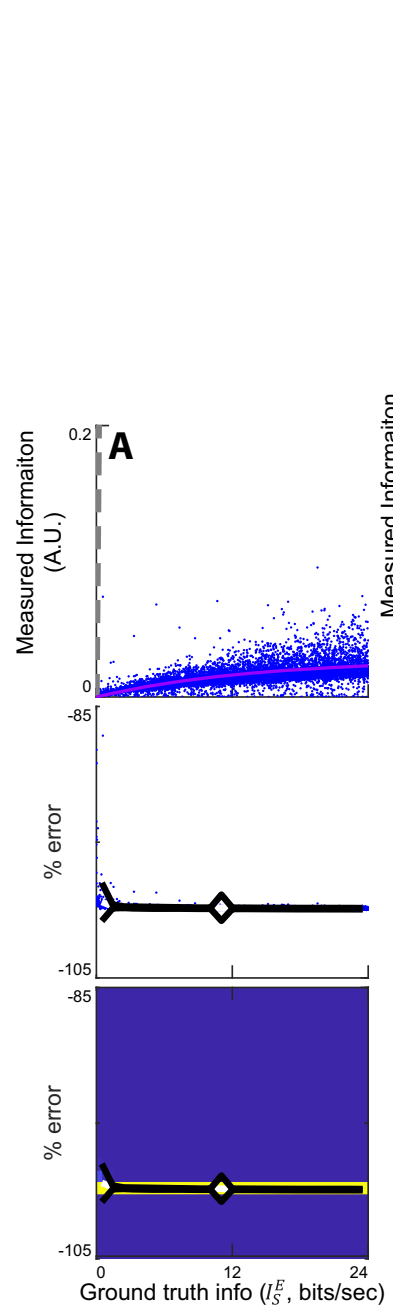
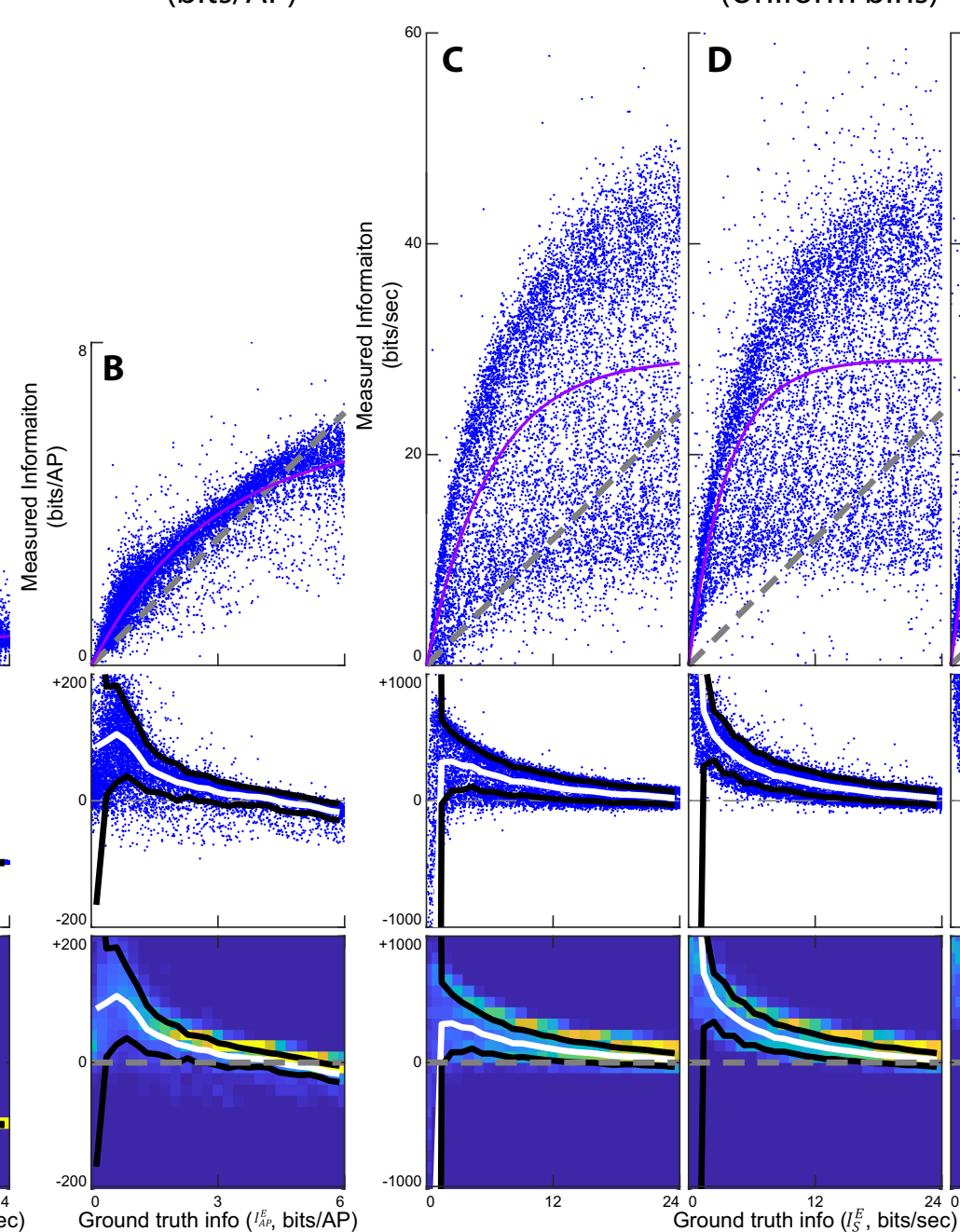

E

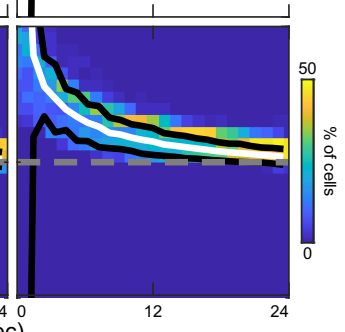

\section{$\mathbf{F}$}

\begin{tabular}{|c|c|c|c|c|c|c|c|c|c|c|}
\hline Method & Units & Source & Mean error & Mean abs error & Mean \% error & Mean abs \% err & C & p exponential & saturation level & $90 \%$ saturation point \\
\hline SMGM bits/sec & bits $\Delta F / F / A P$ & Skaggs et al, 1993 & -11.1 & 11.1 & -96.0 & 96.0 & 0.0390 & 0.760 & 39.7 & $2.3 \mathrm{e} 3$ \\
\hline SMGM bits/AP & bits/AP & Skaggs et al, 1993 & -0.38 & 0.4 & -9.7 & 12.9 & 0.78 & $<<0.01$ & 5.78 & 11.5 \\
\hline foopsi+SMGM bits/sec & $A U$ & Vogelstein et al, 2010 & -11.4 & 11.4 & -99.8 & 99.8 & $1.15 \mathrm{e}-3$ & 0.129 & 0.02 & 32.0 \\
\hline Foopsi+SMGM bits/ap & bits/AP & Vogelstein et al, 2010 & 0.3 & 0.6 & 46.3 & 52.7 & 1.02 & $<<0.01$ & 5.51 & 6.6 \\
\hline KSG & bits/sec & Kraskov et al, 2004 & 9.9 & 11.9 & 37.9 & 254.8 & 1.64 & $<<0.01$ & 29.2 & 13.9 \\
\hline $\begin{array}{c}\text { Binned Ml } \\
\text { (uniform bins) } \\
\text { Binned MI }\end{array}$ & bits/sec & Timm and Lapish, 2018 & 12.8 & 14.1 & 285.7 & 292.5 & 1.77 & $<<0.01$ & 29 & 8.47 \\
\hline (occupancy bins) & bits/sec & Timm and Lapish, 2018 & 15.0 & 15.6 & 322.5 & 325.5 & 1.94 & $<<0.01$ & 32.2 & 9.26 \\
\hline
\end{tabular}

Figure 4. Alternative techniques for measuring $\mathrm{Ml}$ from functional fluorescence traces. $\boldsymbol{A}-\boldsymbol{E}$, top, Information measured from mock GCaMP6f traces versus ground truth information. The gray line is the unity line, the pink line is the best fit saturating exponential. Middle, Percentage error for the information measurements shown on top. Bottom, Heat map of percentage error measurements shown in middle. A, FOOPSI deconvolved traces using the SMGM bits per second metric $\left(l_{\mathrm{s}}^{\mathrm{d}}\right)$. B , FOOPSI deconvolved traces using the SMGM bits per AP metric (l d $\left.I_{A P}\right)$. The regularization coefficient had little effect on these results (Extended Data Figure 4-1). $\boldsymbol{C}$, The KSG measure applied to GCaMP6f traces. $\boldsymbol{D}$, The binned estimator applied to GCaMP6f traces using uniform bins. $\boldsymbol{E}$, The binned estimator applied to GCaMP6f traces using equal occupancy bins. The binned estimators were less distorted on the raw AP traces (Extended Data Figure 4-2). $\boldsymbol{F}$, Table of summary statistics for each measure. $\mathrm{P}$ exponential is the $p$ value from the $\chi^{2}$ test used to determine whether a saturating exponential fit is better than a linear fit for the measured versus ground truth information plots. An analytic solution yielded qualitatively similar, but quantitatively disparate results (Extended Data Figure 4-3). 
either direct application of the SMGM metrics $\left(\hat{l}_{S}^{F}\right.$ and $\left.I_{A P}\right)$ or the application of the SMGM metrics to deconvolved ztraces $\left(l_{s}^{d}\right.$ and $\left.I_{A P}^{\hat{d}}\right)$, we found better recovery using the direct application approach $\left(\hat{l}_{S}^{F}\right.$ and $\left.I_{A P}\right)$.

\section{The KSG and binned estimators are poor estimators of $\mathrm{MI}$ in functional florescence data}

In addition to SMGM, the KSG and binned estimation metrics have been developed for estimating $\mathrm{MI}$ between variables. These other two metrics produce information measured in bits per second, so they are only comparable to the SMGM bits per second estimator $\left(\hat{I}_{s}\right)$. The KSG metric uses the kth nearest neighbor distances between points in the neural response and behavioral variable space to estimate information (Kraskov et al., 2004). The binned estimation metric uses discrete bins to estimate the full multidimensional joint probability distribution $\left(p_{(X, Y)}\right.$ in Eq. 1) to estimate Ml (Timme and Lapish, 2018). These two metrics estimate information across time samples and therefore are dependent on firing rate like the SMGM bits per second metric considered above. Further, the binned estimator is sensitive to the precise method used for data binning, and thus, we have used two commonly applied binning methods: uniform and occupancybased bins.

We applied the KSG, binned estimator (uniform bins), and binned estimator (occupancy binned; Materials and Methods) to the same 10,000 mock GCaMP6f time-series traces and behavioral data used to assess the SMGM approach. These methods all behaved similarly when applied to our simulations (Fig. 4C-E), so they will be discussed together here. The information values measured by these techniques correlated with ground truth information in bits per second $\left(I_{S}^{E}\right)$. Interestingly, unlike the SMGM bits per second metric ( $\left(\hat{I}_{s}\right.$; Fig. 2), the KSG and binned estimator results were better fit with a saturating exponential than with a linear fit $\left(\chi_{1}^{2}=1.70 \mathrm{e} 4,3.12 \mathrm{e} 4\right.$, and $3.14 \mathrm{e} 4$, respectively, $p \sim 0$ ). The KSG and binned estimator methods overestimated the information at lower ground truth $\left(l_{s}^{E}\right)$ values and saturated quickly at higher values. For ground truth information $\left(I_{s}^{E}\right)$ values below 10 bits per second, the mean absolute errors were $10.3 \pm$ 7.82 bits per second ( $465 \pm 4390 \%), 22.2 \pm 12.6$ bits per second $(821.7 \pm 3353 \%)$, and $24.2 \pm 14.0$ bits per second $(911 \pm 3926 \%)$ for the KSG, binned estimator (uniform bins), and binned estimator (occupancy binned), respectively (Fig. 4F). This is in comparison to the $0.35 \pm$ $0.59 \frac{b^{\Delta i t s}{ }^{\Delta F} / F}{A P}(14 \pm 103 \%)$ mean absolute error found using the SMGM bits per second metric $\left(\hat{I}_{s}\right)$ for ground truth information values $<10$ bits per second. For ground truth information $\left(I_{s}^{E}\right)$ values $>10$ bits per second, the mean absolute errors were $13.2 \pm 8.66$ bits per second $(85.0 \pm$ $64.4 \%), 25.4 \pm 16.2$ bits per second (165.2 $\pm 118.3 \%)$, and $28.9 \pm 17.34$ bits per second $(186.3 \pm 124.9 \%)$ for the KSG, binned estimator (uniform bins), and binned estimator (occupancy binned), respectively. This is in comparison to the $0.97 \pm 1.15$ bits per second $(5.85 \pm 6.73 \%)$ mean absolute errors found using the SMGM bits per second metric $\left(\hat{I}_{s}\right)$ for ground truth information values $>10$ bits per second. Over the full range of ground truth values, mean absolute errors of $11.9 \pm 8.42$ bits per second $(254.8 \%), 23.5 \pm 14.8$ bits per second $(456.7 \%)$ and $26.7 \pm 16.1$ bits per second (509.0\%) were found for the KSG, binned estimator (uniform bins), and binned estimator (occupancy binned), respectively, an order magnitude larger than the $2.4 \pm 2.97 \mathrm{AU}(22 \pm 27 \%$ error) error seen using the SMGM bits per second metric $\left(\hat{F}_{s}\right)$. As a control, we applied the binned estimator s to AP traces and compared the estimated information to the ground truth information to verify that the large errors observed (Fig. 4D,E) were caused when the estimators were applied to fluorescence data (rather than simply a difference between the binned estimator, which do not rely on a Poisson firing assumption, and the ground truth information established using SMGM, which does rely on a Poisson firing assumption). We found the errors when applying the binned estimators to AP traces were relatively small [mean absolute error $2.72 \pm 3.38$ bits per second $(41.1 \%)$ and $2.70 \pm 3.33$ bits per second $(41.0 \%)$ for the uniform and occupancy-based binning; respectively; Extended Data Fig. 4-2]. Therefore, when comparing the recovery of ground truth information from functional fluorescence traces using either the SMGM metric $\hat{I}_{s}^{F}$ or the KSG and binned estimator metrics, we found better recovery using the SMGM approach $\left(\hat{I}_{s}\right)$.

\section{An analytic approximation can reproduce some qualitative, but not quantitative, results of the numeric solutions}

Some of the general features of the relationship between ground truth information and fluorescence SMGM metrics can also be seen using an analytic approximation. For example, if we approximate the rate map as a Gaussian firing field with mean rate $\bar{\lambda}$, simplify the kernel approximation to a single exponential with falloff $\tau$, assume constant, normalized movement speed $v$, and assume that the convolution between the kernel and the mean rate map is nearly Gaussian, we can approximate the relationship between the bits per second ground truth information $I_{S}^{E}$ and measured fluorescence information $I_{S}^{F}$ as

$$
I_{S}^{F} \approx-\frac{A v \tau \bar{\lambda} \log \left(4^{\frac{-1 E_{S}}{\lambda}}+2 \pi e v^{2} \tau^{2}\right)}{\log (4)},
$$

and between the bits per AP ground truth information $I_{A P}^{E}$ and measured fluorescence information $I_{A P}^{F}$ as

$$
I_{A P}^{F} \approx-\frac{\log \left(4^{-I_{A P}^{E}}+2 e \pi v^{2} \tau^{2}\right)}{\log (4)} .
$$

Similar to the numerical solution, the analytic approximation provided by these equations (Eqs. 9, 10) predict that the fluorescence bits per second metric is dominated by a prefactor ( $\mathrm{A} \tau \mathrm{V} \lambda^{-}$in the analytical case), and that the 
fluorescence bits per AP metric saturates at larger information values. Our numerical solutions provide more accurate measures for the magnitude of these effects, and for the magnitude of information values themselves, given that they include the more accurate double exponential kernel, signal noise, and the realistic nonstationary speed, position and fluorescence signals. These quantitative differences can be seen in Extended Data Figure 4-3, where we directly compared this analytic approximation to our numerical approach by simulating 10,000 neurons with Gaussian rate maps $(\lambda(x))$ with known (ground truth) information. We found a significant difference in the slope of the bits per second estimator ( $c, 0.041$ vs $0.0036 \frac{\Delta F / F}{\mathrm{~Hz}}$ for the numeric vs analytical, respectively), likely because of the non-stationarities present in behavior and florescence signals. For the fluorescence bits per AP measure, the analytic approximation predicts a large positive bias for ground truth values up to $\sim 4$ bits/AP. This is in contrast to the numeric solution, which has $<10 \%$ error for ground truth information values below 3.12 bits/AP.

\section{Guidelines for application of information metrics to functional fluorescence imaging data}

Taken together, the above results suggest that across the information metrics applied directly to functional fluorescence traces, the SMGM metrics provide the most reliable and interpretable information measurements. We thus suggest the following guidelines for use and interpretation of the SMGM metrics as applied to fluorescence MI metrics $\left(\hat{I}_{s}^{F}\right.$ and $\left.I_{A P}\right)$ defined in Equations 7, 8.

The SMGM bits per second metric $\left(I_{s}^{F}\right)$ is likely attractive to imaging researchers because the units suggest that precise knowledge of AP numbers and times are not required for its use. However, there are several challenges when applying the SMGM bits per second metric to functional fluorescence imaging data. First, the substitution of the change in fluorescence map $(f)$ for the AP firing rate map $(\lambda)$ introduces a change in units, from bits per second to $\frac{\Delta F}{F} \frac{\text { bits }}{A P}$, which is difficult to interpret and relate back to bits per second. Second, the transformation of AP firing rate to change in fluorescence can be approximated by a $c$ scaling factor $(f=c \lambda)$, which is measured in $\frac{\Delta F / F}{H z}$, a quantity that is unknown a priori. If $c$ is not consistent between the neurons of a population of interest, then the information values will be scaled differently and cannot be directly compared (Fig. 2). Since $c$ is dependent on the width and height of the indicator response to a single AP (the kernel), it can vary from neuron to neuron based on difference in indicator expression level, intracellular calcium buffering, and many other factors (Park and Dunlap, 1998; Aponte et al., 2008; Helmchen and Tank, 2015; Greenberg et al., 2018). More research will be needed to measure these parameters (Chen et al., 2012, 2013), and thus $c$, across neurons. Some results suggest that there may be non-trivial amounts of variability within a populations of neurons (Greenberg et al., 2018; Éltes et al., 2019). With the impact of $c$ on the SMGM bits per second metric, and the possible variability of $c$ across a population of neurons, how can researchers properly extract useful measurements of information using $\hat{l}_{s}^{\hat{F}}$ ?

\section{Guideline 1}

First, we note that if experimental measurements reveal small and acceptable variations in $c$ across the neurons of interest, then the information values derived from $\hat{I}_{s}$ can be normalized by this factor to recover information values in units of SMGM bits per second (independent of $\Delta F / F$ ) that can be compared across neurons.

Under the assumption of a consistent kernel, approximations for $c$ for common indicators can be found in Extended Data Figure 2-2.

\section{Guideline 2}

Further, given small variations in $c$ across the neurons of interest, the ratio of $\hat{l}_{s}^{F}$ between neurons in the population provides a meaningful metric for comparisons. For example, such ratios could be used to divide a population of neurons accurately into groups based on their information values (e.g., three quantiles of information) or compare the information values between different functional subtypes of neurons (e.g., between place and non-place cells).

\section{Guideline 3}

The metric can still be useful even if experimental measurements reveal large and unacceptable variations in $c$ across the neurons of interest, or if experimental measurements of $c$ do not exist. In such cases, since it is reasonable to assume that $c$ is consistent in the same neuron over time, comparisons across the same neuron can provide meaningful insights by using a ratio of $\hat{l}_{s}$ measured (from the same neuron) across different conditions. For example, quantifying the neuron-by-neuron ratio of $l_{s}$ between different behavioral states or conditions of an animal or task, such as between goal-directed versus non-goal-directed running down a linear track, would allow researchers to make conclusions such as the following: "The population of neurons in region $Z$ carries $X+/$ $-Y$ times more information during goal-directed than non-goal-directed running."

Therefore, we conclude that with careful consideration of the (known or unknown) variability of the fluorescence response kernel (c), $\hat{l}_{s}$ can be used to extract useful measurements of information, either direct measurements of information across a population of neurons (with known and similar $c$ ), ratios of information between different neurons of a population (with known and similar c) or differences across different conditions within the same neuron (with $c$ unknown or different across neurons).

The SMGM fluorescence bits per AP metric $\left(I_{A P}\right)$ results in the same units as the AP-based metric $I \hat{E}_{A P}$, and therefore may provide imaging researchers with information values that are relatively easy to interpret. However, this similarity in units is somewhat misleading since the number and timing of APs are not directly measured with functional fluorescence traces and the asymmetric and relatively slow dynamics of fluorescence indicators leads to shifting and smoothing of the AP rate map $(\lambda)$. This issue can have the effect of inducing a significant negative 
bias in information measurements, especially at high information values and with functional indicators with wider kernels (Fig. 3). This is the most important factor to consider when determining how researchers can properly extract useful measurements of information using $I_{A P}$. The shifting and smoothing of the AP rate map by fluorescence effectively leads to crosstalk between adjacent spatial bins. Therefore, it is critical to consider the size of the spatial bins in relation to the spatial shift and smoothing induced by the indicator (effectively the kernel plotted in space, rather than time, using the animal's average running velocity to transform from time to space). It is reasonable then to counteract the spatial shift and smoothing effect by using larger bin sizes, but this only works up to a point since larger bins limit the maximum amount of information possible to measure and may negatively bias the information values near this upper limit, even for APbased recordings (Fig. 3B-D). Researchers could potentially optimize the recovery of ground truth information by appropriately selecting bin size for a particular indicator (see Extended Data Fig. 3-2A,B).

In practice, using gCaMP6f and the rodent spatial behavior and spatial bin sizes $(5 \mathrm{~cm})$ used here, our analysis suggests that $I_{A P}$ provides reasonable measurements of information for neurons with values up to 3 bits/AP (Fig. $3 E-G)$, since this is the point where the absolute error exceeds $10 \%$ [comparable to the mean absolute error when measuring information from AP data (8.4\%)]. Equivalent thresholds for other common indicators are shown in Extended Data Fig. 3-1. The error is exacerbated by slower indicators and thus more accurate measurements of information will result from using the fastest, narrowest kernel indicators available, assuming signal-to-noise and detection efficiency are comparable across the different width indicators.

\section{Guideline 4}

We conclude that with careful consideration of the size of the spatial bins in relation to the spatial shift and smoothing induced by the indicator, $I_{A P}$ can be used to extract useful measurements of information, most accurately for neurons with $<3$ bits/AP under recording conditions similar to those considered here.

Previous research quantifying information in bits per AP using $I_{A P}$ have found that the majority of neurons carry information in this range ( $<3$ bits/AP; Knierim et al., 1995; Markus et al., 1995; Lee et al., 2006; Poucet and Sargolini, 2013; Bourboulou et al., 2019), with a few exceptions (Ji and Wilson, 2007). Although these levels are dependent on the number of bins and bin dwell time, $I_{A P}$ should be widely applicable to quantifying information throughout the brain during behavior.

\section{Example: application of information metrics to functional fluorescence imaging data from hippocampus during spatial behavior}

In this section, we demonstrate use of the above guidelines for proper application and interpretation of the SMGM fluorescence MI metrics $\left(\hat{I}_{S}\right.$ and $\left.I_{A P} \hat{F}\right)$ defined in Equations 7,8 . We applied these metrics to functional fluorescence recordings (gCaMP6f) from pyramidal neurons in CA1 of the hippocampus acquired during mouse spatial behavior.

CA1 neurons expressing gCaMP6f (viral transfection, Camk2a promoter) were imaged with two-photon microscopy through a chronic imaging window during mouse navigation along a familiar $1 \mathrm{D}$ virtual linear track, as described previously (Fig. 5A,B; Dombeck et al., 2010; Sheffield et al., 2017; Radvansky and Dombeck, 2018). Eight fields of view from four mice were recorded in eight total sessions (recording duration $8.8 \pm 1.3 \mathrm{~min}$, number of traversals/session: $29 \pm 2.5,3.6 \pm 0.3 \mathrm{laps} / \mathrm{min}, 3-\mathrm{m}-$ long track). From these eight sessions, 1500 neurons were identified from our segmentation algorithm (see Materials and Methods), and analysis was restricted to the 964 neurons that displayed at least one calcium transient on at least $1 / 3$ of the traversals during the session. Among these 964 neurons, 304 (31.5\%) had significant place fields and were thus identified as place cells (see Materials and Methods), while the remaining 660 (68.5\%) did not pass a place field test and were thus identified as non-place cells.

By applying Equation 7 (using 5-cm sized spatial bins), we found a continuum of spatial information values measured by the fluorescence SMGM bits per second metric $\left(\hat{I}_{\mathrm{S}}^{\hat{F}}\right)$ across the 964 CA1 neurons, with an average value of $\hat{I}_{\mathrm{s}}^{\hat{F}}=0.14 \pm 0.0040 \frac{\text { bits }^{\Delta F} / F}{\mathrm{~Hz}}(\mathrm{Fig} .5 \mathrm{C})$. The units for $\mathrm{I}_{\mathrm{s}}^{\hat{F}}$ make direct use and interpretation of these values difficult, however, because these recordings were all from pyramidal neurons in a single area, here for illustrative purposes, we will presume that variations in c (discussed above) across the 964 neurons of interest are small and acceptable, with the absolute value of $c$ unknown. This allows for comparisons of information ratios across different subsets of the population. For example, place cells had 2.8 \pm 0.20 times more information than nonplace cells using the SMGM bits per second metric $\left(l_{\mathrm{s}}^{\hat{F}}=0.18 \pm 0.0047\right.$ for place vs $0.063 \pm 0.0029 \frac{\mathrm{bits}^{\Delta \mathrm{F}} / \mathrm{F}}{\mathrm{Hz}}$ for non-place, rank-sum $p=1.7 e-63$; Fig. 5D-F), although there was substantial overlap in information between the populations (see distributions in Fig. 5D and individual examples in Fig. $5 E, F$ ). This also allows for accurate division of the 964 neurons into three quantiles based on information values, which we use below for spatial location decoding.

By applying Equation 8 (using $5-\mathrm{cm}$ sized spatial bins), we found a continuum of spatial information values measured by the fluorescence SMGM bits per AP metric $\left(\mathrm{I}_{\mathrm{AP}}^{\mathrm{F}}\right)$ across the 964 CA1 neurons, with an average value of $\mathrm{I}_{\mathrm{AP}}^{\hat{F}}=1.65 \pm 0.023$ bits/AP (Fig. $5 \mathrm{C}$ ). The units for $\mathrm{I}_{\mathrm{AP}}^{\hat{\mathrm{F}}}$ allow for direct use and interpretation of these values, and notably, because most (97\%) of the neurons had values $<3$ bits/AP, a mean absolute error of $<10 \%$ can be assumed across the distribution of SMGM bits per AP values. When applied to the place and non-place cell populations, we found that place cells had higher information than nonplace cells using the fluorescence SMGM bits per AP metric $\left(I_{A P}=1.8 \pm 0.026\right.$ and $1.35 \pm 0.042$ bits/AP, rank-sum 


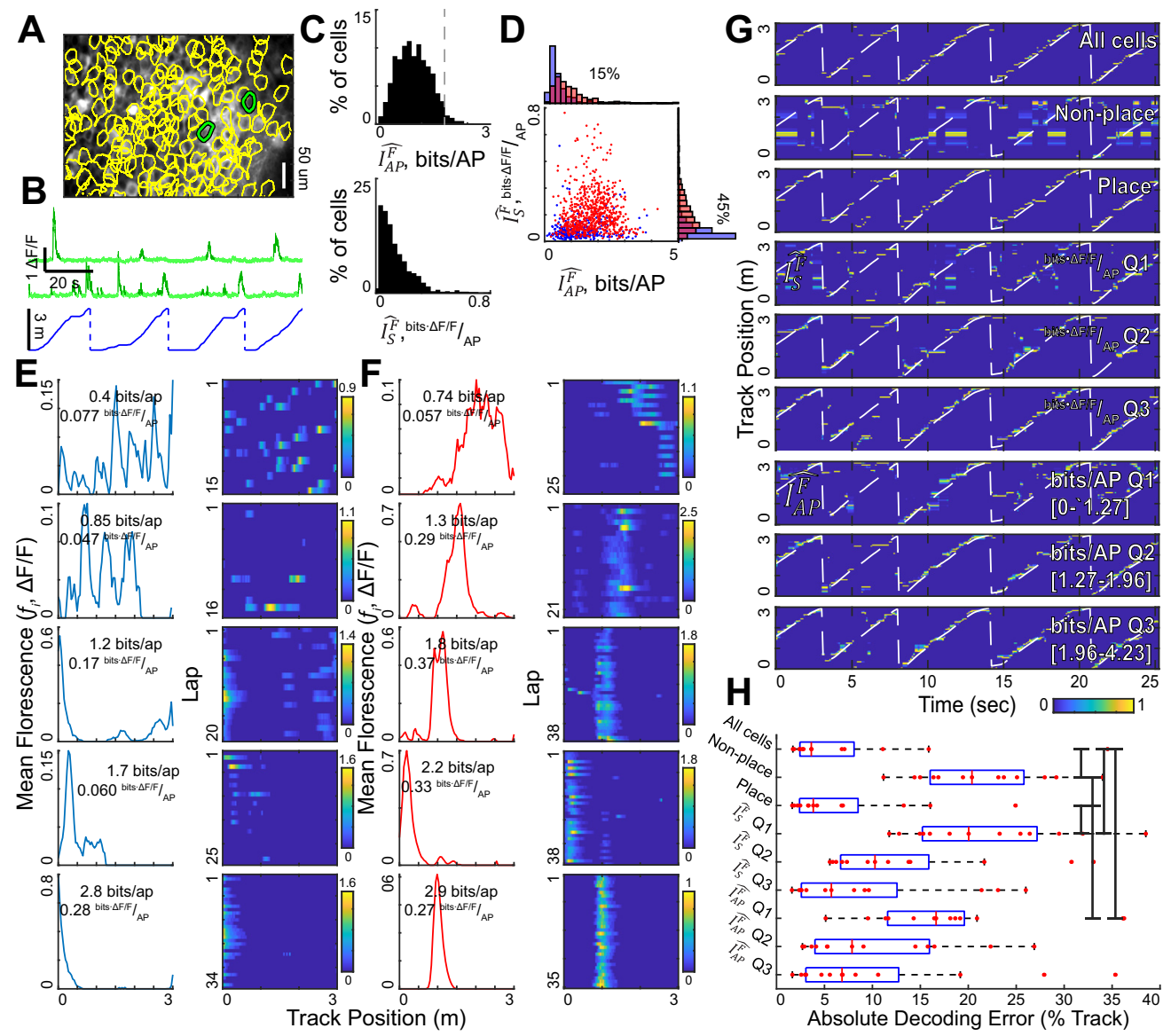

Figure 5. Application of SMGM information metrics to functional fluorescence imaging data from hippocampus during spatial behavior. $\boldsymbol{A}$, Example field of hippocampal pyramidal neurons expressing GCaMP6f and imaged during linear track navigation. Active cell ROls shown in yellow; traces for green cells shown in $\boldsymbol{B}$. $\boldsymbol{B}$, Fluorescence DF/F traces (green) from two neurons in the field shown in $\boldsymbol{A}$ and the track position during the recording (blue). $\boldsymbol{C}$, Distribution of information values using the fluorescence SMGM bits per second metric ( $\left(\hat{I}_{S}^{F}\right.$, top) and the fluorescence SMGM bits per AP metric $\left(I_{A P}^{F}\right.$, bottom). The gray line indicates the recommended cutoff for reliability using GCaMP6f. $D$, Plot of $\hat{I}_{s}$ versus $I_{A P}$ for each neuron. Place cells indicated in red and nonplace cells in blue. $\boldsymbol{E}$, Example non-place cells spanning the information ranges shown in $\boldsymbol{C}$. Spatial fluorescence map $\left(f_{i}\right)$ shown on left, and average change in fluorescence per track traversal on right. $\boldsymbol{F}$, Same as $\boldsymbol{E}$, but for place cells. $\boldsymbol{G}$, Bayesian decoding of mouse's track position using different subpopulations of neurons for one example session. From top to bottom, All active neurons, all nonplace cells, and place cells, the first through third quantiles of the SMGM bits per second formulation $\left(I_{s}^{F}\right)$, and the first through third quantiles of the SMGM bits per AP formation $\left(I_{A P}^{F}\right)$. The white dashed line indicates the ground truth position of the animal, the color map indicates the decoded position probability (peak-normalized posterior distribution). $\boldsymbol{H}$, Decoding accuracy (absolute position decoding error in units of \% of track) pooled over all sessions for each neuron group indicated in G. Black bars indicate significant differences by Holm-Bonferroni corrected rank-sum tests $(\alpha=0.05)$. Consistent results were obtained when measuring information from real spiking data and simulated florescence traces (Extended Data Fig. 5-1).

$p=4.6 \mathrm{e}-21$; Fig. $5 D-F$ ). This is consistent with mock fluorescence traces generated from real neuron AP datasets (Extended Data Fig. 5-1B).

As a demonstration of the usefulness of using information metrics to analyze large functional fluorescence population recordings, we explored the accuracy of decoding the animal's track position using different subsets of neurons. We divided the 964 neurons into nine groups: all neurons, place cells, non-place cells, three quantiles based on the fluorescence SMGM bits per second metric, and three quantiles based on the fluorescence SMGM bits per AP metric. We then used a Bayesian decoder of the animals' position (see Materials and Methods) separately for each of the nine neuron groups in each of the eight sessions (Fig. 5G,H). An individual session decoding example can be seen in Figure 5G. We quantified decoding accuracy using the absolute position decoding error (\% of track), and pooled this measure across sessions for each neuron group (Fig. 5H). The means and standard errors for each group are: all neurons $(7.33 \pm 2.5 \%)$, place cells $(6.97 \pm 1.9 \%)$, non-place cells $(20.9 \pm 1.8 \%)$, SMGM bits per second Q1 (21.9 $\pm 1.5 \%)$, SMGM bits per second Q2 (13.2 $\pm 2.4 \%)$, SMGM bits per second Q3 $(8.97 \pm 2.4 \%)$, SMGM bits per AP Q1 (17.6 $\pm 2.7 \%)$, SMGM bits per AP Q2 $(17.8 \pm 3.1 \%)$, SMGM bits per AP Q3 (10.4 $\pm 3.0 \%)$. Interestingly, even the lowest quantile information groups still could be used to determine animal track location to within $\sim 1 / 5$ of the track. This supports 
the idea that the hippocampal code for space is carried by a large population of active neurons (Meshulam et al., 2016), and not just by a select subpopulation with the highest information or most well-defined tuning curves. As could be expected, place cells encoded the position of the animal better than nonplace cells and better than the lowest quantile information groups (Holm-Bonferroni corrected rank-sum, $\alpha=0.05)$, and neurons in the higher quantiles provided more accurate decoding. Thus, the fluorescence information metrics provide a means to compare the relative contribution of hippocampal neurons with different information values to decoding animal position.

\section{Discussion}

Here, we performed an in-depth simulation study to examine the application of the SMGM bits per second and SMGM bits per AP metrics of MI to functional fluorescence recordings. Since these metrics were designed for AP recordings and since functional fluorescence recordings violate some of the assumptions that these metrics are based on, it was unclear whether and how the metrics could be used for functional fluorescence recordings. We created a library of ten thousand mock neurons whose AP output carried ground-truth amounts of information about the animal's spatial location, and by using real behavioral recording data from mice navigating in virtual linear tracks, we simulated the spatial firing patterns of the mock neurons. We then simulated fluorescent calcium responses for each neuron in each session by convolving the AP trains with calcium kernels for different indicators, primarily GCamp6f (although see Extended Data Figs. 2-2, 3-1 for results from other indicators), and then added noise.

We then derived fluorescence versions of the SMGM bits per second $\left(\hat{I}_{S}\right)$ and SMGM bits per AP metrics $\left(I_{A P}\right.$; Eqs. 7,8$)$ and applied them to the fluorescence traces to quantify the performance of the metrics for estimating information. We found that ground truth information, as measured by the fluorescence SMGM bits per second metric $\left(\hat{I}_{s}\right)$, was transformed into different units and was linearly scaled by a factor (c) dependent on the height and width of the kernel, with $c$ linearly dependent on height and nonlinearly dependent on width. The error induced by these transformations changed substantially over the range of kernel values of the different functional indicators widely used today, and therefore are important factors to consider when designing and interpreting functional imaging experiments. We then found that ground truth information, as measured by the fluorescence SMGM bits per AP metric $\left(I_{A P}\right)$, retains the units and insensitivity to height scaling of the electrophysiological metric $\left(I_{A P}\right)$, but is nonlinearly biased by the smoothing of the fluorescence map dictated by the width of the kernel. The estimation errors strongly depended on both the width of the kernel and the information value being measured. Importantly, since these parameters change substantially over the different functional indicators and different neuron types and behaviors that are commonly used today, they are important factors to consider when designing and interpreting functional imaging experiments. For example, even for the same indicator, the shape of the kernel is a function of intracellular calcium buffering, indicator concentration, the amount of calcium influx, the efflux rates, background fluorescence and resting calcium concentration, which can all vary across different cells. Additionally, the results presented here rely on the approximation that $\Delta F / F$ scales linearly with the firing rate, which is not strictly true in practice. We show in Extended Data Figure 3-3 that even a relatively simple nonlinearity between $\Delta F / F$ and firing rate can add distortions to the amount of information measured using the fluorescence SMGM approach. This relationship between $\Delta F / F$ and firing rate can vary across different indicators and, since the Toolbox can be used to vary this relationship, users can further explore this source of bias.

In our approach, the known information values in our library of 10,000 mock neurons were determined using the SMGM metric, which includes the assumption that neuron firing follows an inhomogeneous Poisson process. It is important to remember that the SMGM metric, which has been applied to spiking data extensively over the past few decades, requires the use of a Poisson estimate of spiking probability, i.e., the Poisson assumption is built into the original metric. In practice, even spiking data violates this and other assumptions of the SMGM metric since real neurons do not strictly follow Poisson statistics (for example, they can display neural hysteresis) and animal behavior is non-stationary. Here, we are building from this existing framework and adding and testing whether it is possible to apply the metric to functional fluorescence datasets. Even still, the Poisson assumption could have contributed to some of the biases found when evaluating the fluorescence SMGM metrics with respect to ground truth information. We explored this potential source of bias further using two different analyses. First, in Extended Data Figure 4-2, we applied the binned estimators (which do not rely on a Poisson firing assumption) to AP traces and compared the estimated information to the ground truth information (which was established using the SMGM metric that does rely on a Poisson firing assumption). We found the errors to be relatively small, particularly in comparison to the errors induced by the binned estimators when applied to fluorescence traces (Fig. 4D,E). Second, in Extended Data Figure 5-1, we used a real spiking dataset from hippocampal neurons in mice running on a behavioral track (i.e., real spiking neurons that can deviate from Poisson firing) and generated mock fluorescence traces from the AP traces. When we compared the information measured from the AP traces to the fluorescence traces, we found biases that were largely consistent with those observed in Figures 2, 3 from our simulated mock neuron datasets. Taken together, these analyses indicate that any biases resulting from the Poisson assumption in the simulation procedure appear to be small, particularly with respect to the biases introduced when AP traces are transformed into functional fluorescence traces. Finally, in 
the Toolbox, we also include code to generate mock neurons using a binned distribution, avoiding the Poisson assumption of SMGM. Thus, users can further explore sources of bias using a different ground truth dataset.

Using our mock fluorescence traces, we also asked whether an AP estimation method could relieve the biases in the SMGM metrics. Applying the SMGM bits per second metric $\left(l_{s}^{d}\right)$ to AP estimation traces from a deconvolution algorithm (FOOPSI) resulted in a low $c$ value for recovered versus ground truth information. When the SMGM bits per AP measure was applied $\left(l_{A P}^{\hat{d}}\right)$, the resulting measurements of information were still nonlinear (compared with $l_{s}$ ), with a positive bias at lower values of ground truth information. Overall, applying FOOPSI to fluorescence traces led to a poorer recovery of ground truth information using SMGM compared with direct application of SMGM to the florescence traces $\left(\hat{I}_{A P}\right)$. Importantly, this result from deconvolution is only specific to GCaMP6f, and conclusions should not be drawn about other indicators or situations; users will be able to use the Toolbox to explore this area further. We also tested other metrics to measure Ml directly from the fluorescence time traces [KSG, binned estimator (uniform bins), and binned estimator (occupancy binned)] and found these alternatives produced highly variable, saturating measurements of recovered versus ground truth information. This was in contrast to the SMGM bits per second measure $\left(\hat{I}_{s}\right)$ which produced a linearly scaled bias with lower error.

Taken together, we find that the SMGM bits per AP metric can well recover the MI between spiking and behavior. The SMGM bits per second metric is scaled such that comparisons should be limited to within populations of well characterized neurons or for within neuron comparisons, e.g., ratios of information across conditions. In general, researchers should use caution when applying measures developed for AP data in fluorescence recordings: there's no guarantee that the assumptions that support the measures hold for fluorescence data, and this can lead to difficult to interpret and biased results.

\section{References}

Adoff MD, Climer JR, Davoudi H, Marvin JS, Looger LL, Dombeck DA (2021) The functional organization of excitatory synaptic input to place cells. Nat Commun 12:3558.

Ahrens MB, Orger MB, Robson DN, Li JM, Keller PJ (2013) Wholebrain functional imaging at cellular resolution using light-sheet microscopy. Nat Methods 10:413-420.

Allen TA, Salz DM, McKenzie S, Fortin NJ (2016) Nonspatial sequence coding in CA1 neurons. J Neurosci 36:1547-1563.

Aponte Y, Bischofberger J, Jonas P (2008) Efficient Ca2+ buffering in fast-spiking basket cells of rat hippocampus. J Physiol 586:2061-2075.

Aronov D, Tank DW (2014) Engagement of neural circuits underlying $2 \mathrm{D}$ spatial navigation in a rodent virtual reality system. Neuron 84:442-456.

Belghazi MI, Baratin A, Rajeswar S, Ozair S, Bengio Y, Courville A, Hjelm RD (2018) MINE: mutual information neural estimation. 35th Int Conf Mach Learn ICML 2018 2:864-873.
Bourboulou R, Marti G, Michon FX, El Feghaly E, Nouguier M, Robbe D, Koenig J, Epsztein J (2019) Dynamic control of hippocampal spatial coding resolution by local visual cues. Elife 8:e44487.

Brandon MP, Bogaard AR, Libby CP, Connerney MA, Gupta K, Hasselmo ME (2011) Reduction of theta rhythm dissociates grid cell spatial periodicity from directional tuning. Science 332:595599.

Brown EN, Barbieri R, Eden UT, Frank L (2003) Likelihood methods for neural spike train data analysis. In: Computational neuroscience: a comprehensive approach (Feng J, ed), pp 252-281. Boca Raton: CRC Press.

Buzsáki G, Mizuseki K (2014) The log-dynamic brain: how skewed distributions affect network operations. Nat Rev Neurosci 15:264278.

Calton JL, Stackman RW, Goodridge JP, Archey WB, Dudchenko PA, Taube JS (2003) Hippocampal place cell instability after lesions of the head direction cell network. J Neurosci 23:97199731.

Chen Q, Cichon J, Wang W, Qiu L, Lee SJR, Campbell NR, DeStefino N, Goard MJ, Fu Z, Yasuda R, Looger LL, Arenkiel BR, Gan WB, Feng G (2012) Imaging neural activity using Thy1GCaMP transgenic mice. Neuron 76:297-308.

Chen TW, Wardill TJ, Sun Y, Pulver SR, Renninger SL, Baohan A, Schreiter ER, Kerr RA, Orger MB, Jayaraman V, Looger LL, Svoboda K, Kim DS (2013) Ultrasensitive fluorescent proteins for imaging neuronal activity. Nature 499:295-300.

Chen Z, Grosmark AD, Penagos H, Wilson MA (2016) Uncovering representations of sleep-associated hippocampal ensemble spike activity. Sci Rep 6:32193.

Climer JR, Newman EL, Hasselmo ME (2013) Phase coding by grid cells in unconstrained environments: two-dimensional phase precession. Eur J Neurosci 38:2526-2541.

Climer JR, DiTullio R, Newman EL, Hasselmo ME, Eden UT (2015) Examination of rhythmicity of extracellularly recorded neurons in the entorhinal cortex. Hippocampus 25:460-473.

Cohen MR, Kohn A (2011) Measuring and interpreting neuronal correlations. Nat Neurosci 14:811-819.

Dana H, Sun Y, Mohar B, Hulse B, Hasseman JP, Tsegaye G, Tsang A, Wong A, Patel R, Macklin JJ, Chen Y, Konnerth A, Jayaraman V, Looger LL, Schreiter ER, Svoboda K, Kim DS, H, Dana Y, Sun B, Mohar B, et al. (2018) High-performance GFP-based calcium indicators for imaging activity in neuronal populations and microcompartments. bioRxiv 16:434589.

Dana H, Sun $Y$, Mohar B, Hulse BK, Kerlin AM, Hasseman JP, Tsegaye G, Tsang A, Wong A, Patel R, Macklin JJ, Chen $Y$, Konnerth A, Jayaraman V, Looger LL, Schreiter ER, Svoboda K, Kim DS (2019) High-performance calcium sensors for imaging activity in neuronal populations and microcompartments. Nat Methods 16:649-657.

DeWeese MR, Zador AM, Hromádka T (2008) Sparse representation of sounds in the unanesthetized auditory cortex. PLoS Biol 6:e16.

Dombeck DA, Khabbaz AN, Collman F, Adelman TL, Tank DW (2007) Imaging large-scale neural activity with cellular resolution in awake, mobile mice. Neuron 56:43-57.

Dombeck DA, Harvey CD, Tian L, Looger LL, Tank DW (2010) Functional imaging of hippocampal place cells at cellular resolution during virtual navigation. Nat Neurosci 13:1433-1440.

Éltes T, Szoboszlay M, Kerti-Szigeti K, Nusser Z (2019) Improved spike inference accuracy by estimating the peak amplitude of unitary $\left[\mathrm{Ca}^{2+}\right]$ transients in weakly GCaMP6f-expressing hippocampal pyramidal cells. J Physiol 597:2925-2947.

Evans MH, Petersen RS, Humphries MD (2019) On the use of calcium deconvolution algorithms in practical contexts. bioRxiv 871137.

Frank LM, Brown EN, Wilson MA (2001) A comparison of the firing properties of putative excitatory and inhibitory neurons from CA1 and the entorhinal cortex. J Neurophysiol 86:20292040. 
Friedrich J, Zhou P, Paninski L (2017) Fast online deconvolution of calcium imaging data. PLoS Comput Biol 13:e1005423.

Fu H, Rodriguez GA, Herman M, Emrani S, Nahmani E, Barrett G, Figueroa HY, Goldberg E, Hussaini SA, Duff KE (2017) Tau pathology induces excitatory neuron loss, grid cell dysfunction, and spatial memory deficits reminiscent of early Alzheimer's disease. Neuron 93:533-541.e5.

Fyhn M, Molden S, Hollup S, Moser M-B, Moser El (2002) Hippocampal neurons responding to first-time dislocation of a target object. Neuron 35:555-566.

Gao W, Kannan S, Oh S, Viswanath P (2017) Estimating mutual information for discrete-continuous mixtures. Advances in neural information processing systems, pp 5987-5998. Curren Associates, Inc: Long Beach California.

Gerrard JL, Burke SN, McNaughton BL, Barnes CA (2008) Sequence reactivation in the hippocampus is impaired in aged rats. $J$ Neurosci 28:7883-7890.

Greenberg DS, Wallace DJ, Voit K-M, Wuertenberger S, Czubayko U, Monsees A, Handa T, Vogelstein JT, Seifert R, Groemping Y, Kerr JN (2018) Accurate action potential inference from a calcium sensor protein through biophysical modeling. bioRxiv 479055.

Grosmark AD, Buzsáki G (2016) Diversity in neural firing dynamics supports both rigid and learned hippocampal sequences. Science 351:1440-1443.

Grossmark AD, Long J, Buzsáki G (2016) Recordings from hippocampal area CA1, PRE, during and POST novel spatial learning. doi: 10.6080/K0862DC5.

Grubb MS, Thompson ID (2003) Quantitative characterization of visual response properties in the mouse dorsal lateral geniculate nucleus. J Neurophysiol 90:3594-3607.

Hainmueller T, Bartos M (2018) Parallel emergence of stable and dynamic memory engrams in the hippocampus. Nature 558:292296.

Harvey CD, Collman F, Dombeck DA, Tank DW (2009) Intracellular dynamics of hippocampal place cells during virtual navigation. Nature 461:941-946.

Hazama Y, Tamura R (2019) Effects of self-locomotion on the activity of place cells in the hippocampus of a freely behaving monkey. Neurosci Lett 701:32-37.

Helmchen F, Tank DW (2015) A single-compartment model of calcium dynamics in nerve terminals and dendrites. Cold Spring Harb Protoc 2015:155-167.

Heys JG, Dombeck DA (2018) Evidence for a subcircuit in medial entorhinal cortex representing elapsed time during immobility. Nat Neurosci 21:1574-1582.

Heys JG, Rangarajan KV, Dombeck DA (2014) The functional microorganization of grid cells revealed by cellular-resolution imaging. Neuron 84:1079-1090.

Hinman JR, Brandon MP, Climer JR, Chapman GW, Hasselmo ME (2016) Multiple running speed signals in medial entorhinal cortex. Neuron 91:666-679.

Hok V, Chah E, Save E, Poucet B (2013) Prefrontal cortex focally modulates hippocampal place cell firing patterns. J Neurosci 33:3443-3451.

Huang Y, Brandon MP, Griffin AL, Hasselmo ME, Eden UT (2009) Decoding movement trajectories through a T-maze using point process filters applied to place field data from rat hippocampal region CA1. Neural Comput 21:3305-3334.

Hubel DH, Wiesel TN (2009) Republication of The $\mathrm{J}$ of Physiology (1959) 148, 574-591: receptive fields of single neurones in the cat's striate cortex. 1959. J Physiol 587:2721-2732.

Ji D, Wilson MA (2007) Coordinated memory replay in the visual cortex and hippocampus during sleep. Nat Neurosci 10:100107.

Jing M, Zhang P, Wang G, Feng J, Mesik L, Zeng J, Jiang $H$, Wang S, Looby JC, Guagliardo NA, Langma LW, Lu J, Zuo Y, Talmage DA, Role LW, Barrett PQ, Zhang LI, Luo M, Song Y, Zhu JJ, et al. (2018a) Dendritic inhibition in the hippocampus supports fear learning. Nat Biotechnol 36:726-737.
Jing $M$, Zhang $P$, Wang $G$, Feng J, Mesik L, Zeng J, Jiang $H$, Wang S, Looby JC, Guagliardo NA, Langma LW, Lu J, Zuo Y, Talmage DA, Role LW, Barrett PQ, Zhang LI, Luo M, Song Y, Zhu JJ, et al. (2018b) State-dependent subnetworks of parvalbumin-expressing interneurons in neocortex. Nat Biotechnol 36:726-737.

Jing $M$, Zhang $P$, Wang $G$, Feng J, Mesik L, Zeng J, Jiang $H$, Wang S, Looby JC, Guagliardo NA, Langma LW, Lu J, Zuo Y, Talmage DA, Role LW, Barrett PQ, Zhang LI, Luo M, Song Y, Zhu JJ, et al. (2018c) Calcium dynamics in dendrites of hippocampal CA1 interneurons in awake mice. Nat Biotechnol 36:726-737.

Jing $M$, Zhang $P$, Wang $G$, Feng J, Mesik L, Zeng J, Jiang $H$, Wang S, Looby JC, Guagliardo NA, Langma LW, Lu J, Zuo Y, Talmage DA, Role LW, Barrett PQ, Zhang LI, Luo M, Song Y, Zhu JJ, et al. (2018d) A genetically encoded fluorescent acetylcholine indicator for in vitro and in vivo studies. Nat Biotechnol 36:726-737.

Kalko EV, Dukas R, Ratcliffe JM, Teeling EC, Haven N, Fattu JM, Bates ME, Simmons JA, Riquimaroux H, Surlykke A, Bouffard FH, Lee DN, Dear SP, Horiuchi TK, Krishnaprasad PS, Moss CF, Schuller G, Brudzynski SM, Syme DA, Hollingworth S, et al. (2011) An expanded palette of genetically encoded $\mathrm{Ca} 2+$ indicators. Science 333:1888-1891.

Keller PJ, Ahrens MB (2015) Visualizing whole-brain activity and development at the single-cell level using light-sheet microscopy. Neuron 85:462-483.

Khoshkhoo S, Vogt D, Sohal VS (2017) Dynamic, cell-type-specific roles for GABAergic interneurons in a mouse model of optogenetically inducible seizures. Neuron 93:291-298.

Kinsky NR, Sullivan DW, Mau W, Hasselmo ME, Eichenbaum HB (2018) Hippocampal place fields maintain a coherent and flexible map across long timescales. Curr Biol 28:3578-3588.e6.

Knierim JJ, Kudrimoti HS, McNaughton BL (1995) Place cells, head direction cells, and the learning of landmark stability. J Neurosci 15:1648-1659.

Koenig J, Linder AN, Leutgeb JK, Leutgeb S (2011) The spatial periodicity of grid cells is not sustained during reduced theta oscillations. Science 332:592-595.

Kraskov A, Stögbauer H, Grassberger P (2004) Estimating mutual information. Phys Rev E Stat Physics Plasmas Fluids Relat Interdiscip Top 69:16.

Kraus BJ, Brandon MP, Robinson RJ, Connerney MA, Hasselmo ME, Eichenbaum HB (2015) During running in place, grid cells integrate elapsed time and distance run. Neuron 88: 578-589.

Kropff E, Carmichael JE, Moser M-B, Moser El (2015) Speed cells in the medial entorhinal cortex. Nature 523:419-424.

Lee I, Griffin AL, Zilli EA, Eichenbaum HB, Hasselmo ME (2006) Gradual translocation of spatial correlates of neuronal firing in the hippocampus toward prospective reward locations. Neuron 51:639-650.

Leveteau J, MacLeod P (1966) Olfactory discrimination in the rabbit olfactory glomerulus. Science 153:175-176.

Liu P, Jerrard LE, Bilkey DK, Jarrard LE, Bilkey DK (2004) Excitotoxic lesions of the pre- and parasubiculum disrupt the place fields of hippocampal pyramidal cells. Hippocampus 14:107-116.

MacDonald CJ, Carrow S, Place R, Eichenbaum HB (2013) Distinct hippocampal time cell sequences represent odor memories in immobilized rats. J Neurosci 33:14607-14616.

Mankin EA, Thurley K, Chenani A, Haas OV, Debs L, Henke J, Galinato M, Leutgeb JK, Leutgeb S, Leibold C (2019) The hippocampal code for space in Mongolian gerbils. Hippocampus 29:787-715.

Mann K, Gallen CL, Clandinin TR (2017) Whole-brain calcium imaging reveals an intrinsic functional network in Drosophila. Curr Biol 27:2389-2396.e4.

Markus EJ, Qin YL, Leonard B, Skaggs WEW, McNaughton BBL, Barnes CA (1995) Interactions between location and task affect 
the spatial and directional firing of hippocampal neurons. J Neurosci 15:7079-7094.

Marvin JS, Scholl B, Wilson DE, Podgorski K, Kazemipour A, Müller JA, Schoch S, Quiroz FJU, Rebola N, Bao H, Little JP, Tkachuk AN, Cai E, Hantman AW, Wang SSH, DePiero VJ, Borghuis BG, Chapman ER, Dietrich D, DiGregorio DA, et al. (2018) Stability, affinity, and chromatic variants of the glutamate sensor iGluSnFR. Nat Methods 15:936-939.

Marvin JS, Shimoda Y, Magloire V, Leite M, Kawashima T, Jensen TP, Kolb I, Knott EL, Novak O, Podgorski K, Leidenheimer NJ, Rusakov DA, Ahrens MB, Kullmann DM, Looger LL (2019) A genetically encoded fluorescent sensor for in vivo imaging of GABA. Nat Methods 16:763-770.

Mau W, Sullivan DW, Kinsky NR, Hasselmo ME, Howard MW, Eichenbaum H (2018) The same hippocampal CA1 population simultaneously codes temporal information over multiple timescales. Curr Biol 28:1499-1508.e4.

Meshulam L, Gauthier JL, Brody CD, Tank DW, Bialek W (2016) Collective behavior of place and non-place neurons in the hippocampal network. Neuron 96:1178-1192.e4.

Miri A, Daie K, Burdine RD, Aksay E, Tank DW (2011) Regressionbased identification of behavior-encoding neurons during largescale optical imaging of neural activity at cellular resolution. $J$ Neurophysiol 105:964-980.

Morris TP, White IR, Crowther MJ (2019) Using simulation studies to evaluate statistical methods. Stat Med 38:2074-2102.

Mukamel EA, Nimmerjahn A, Schnitzer MJ (2009) Automated analysis of cellular signals from large-scale calcium imaging data. Neuron 63:747-760.

Newman EL, Climer JR, Hasselmo ME (2014) Grid cell spatial tuning reduced following systemic muscarinic receptor blockade. Hippocampus 24:643-655.

Nguyen MN, Hori E, Matsumoto J, Tran AH, Ono T, Nishijo H (2013) Neuronal responses to face-like stimuli in the monkey pulvinar. Eur J Neurosci 37:35-51.

Nguyen MN, Matsumoto J, Hori E, Maior RS, Tomaz C, Tran AH, Ono T, Nishijo H (2014) Neuronal responses to face-like and facial stimuli in the monkey superior colliculus. Front Behav Neurosci 8:85.

Nguyen JP, Shipley FB, Linder AN, Plummer GS, Liu M, Setru SU, Shaevitz JW, Leifer AM (2016) Whole-brain calcium imaging with cellular resolution in freely behaving Caenorhabditis elegans. Proc Natl Acad Sci USA 113:E1074-E1081.

Niell CM, Stryker MP (2008) Highly selective receptive fields in mouse visual cortex. J Neurosci 28:7520-7536.

O'Connor DH, Peron SP, Huber D, Svoboda K (2010) Neural activity in barrel cortex underlying vibrissa-based object localization in mice. Neuron 67:1048-1061.

O'Keefe J (1976) Place units in the hippocampus of the freely moving rat. Exp Neurol 51:78-109.

Østergaard J, Kramer MA, Eden UT (2018) Capturing spike variability in noisy Izhikevich neurons using point process generalized linear models. Neural Comput 30:125-148.

Pachitariu M, Stringer C, Harris KD (2018) Robustness of spike deconvolution for neuronal calcium imaging. J Neurosci 38:79767985.

Paninski L (2004) Maximum likelihood estimation of cascade pointprocess neural encoding models. Netw Comput Neural Syst 15:243-262.

Park D, Dunlap K (1998) Dynamic regulation of calcium influx by Gproteins, action potential waveform, and neuronal firing frequency. J Neurosci 18:6757-6766.

Park E, Dvorak D, Fenton AA (2011) Ensemble place codes in hippocampus: CA1, CA3, and dentate gyrus place cells have multiple place fields in large environments. PLoS One 6:e22349.

Park IJ, Bobkov YV, Ache BW, Principe JC (2013) Quantifying bursting neuron activity from calcium signals using blind deconvolution. J Neurosci Methods 218:196-205.
Pastalkova E, Itskov V, Amarasingham A, Buzsáki G (2008) Internally generated cell assembly sequences in the rat hippocampus. Science 321:1322-1327.

Pologruto TA, Sabatini BL, Svoboda K (2003) Scanlmage: flexible software for operating laser scanning microscopes. Biomed Eng Online 2:13.

Poucet B, Sargolini F (2013) A trace of your place. Science 340:3536.

Radvansky BA, Dombeck DA (2018) An olfactory virtual reality system for mice. Nat Commun 9:839.

Rashid SK, Pedrosa V, Dufour MA, Moore JJ, Chavlis S, Delatorre RG, Poirazi P, Clopath C, Basu J (2020) The dendritic spatial code: branch-specific place tuning and its experience-dependent decoupling. bioRxiv 2020.01.24.916643.

Robbe D, Buzsáki G (2009) Alteration of theta timescale dynamics of hippocampal place cells by a cannabinoid is associated with memory impairment. J Neurosci 29:12597-12605.

Roxin A, Brunel N, Hansel D, Mongillo G, van Vreeswijk C (2011) On the distribution of firing rates in networks of cortical neurons. $J$ Neurosci 31:16217-16226.

Scholl B, Wilson DE, Fitzpatrick D (2017) Local order within global disorder: synaptic architecture of visual space. Neuron 96:11271138.e4.

Shafi M, Zhou Y, Quintana J, Chow C, Fuster J, Bodner M (2007) Variability in neuronal activity in primate cortex during working memory tasks. Neuroscience 146:1082-1108.

Sheffield MEJ, Dombeck DA (2015) Calcium transient prevalence across the dendritic arbour predicts place field properties. Nature 517:200-204.

Sheffield MEJ, Adoff MD, Dombeck DA (2017) Increased prevalence of calcium transients across the dendritic arbor during place field formation. Neuron 96:490-504.e5.

Simonnet J, Brecht M (2019) Burst firing and spatial coding in subicular principal cells. J Neurosci 39:3651-3662.

Skaggs WE, McNaughton BL, Gothard KM (1993) An informationtheoretic approach to deciphering the hippocampal code 10301037. In: Advances in neural information processing systems 5 (Hanson SJ, Cowan JD, Giles CL, eds). Burlington: MorganKaufmann.

Soo FS, Schwartz GW, Sadeghi K, Berry MJ (2011) Fine spatial information represented in a population of retinal ganglion cells. $J$ Neurosci 31:2145-2155.

Stackman RW, Taube JS (1998) Firing properties of rat lateral mammillary single units: head direction, head pitch, and angular head velocity. J Neurosci 18:9020-9037.

Stirman JN, Smith IT, Kudenov MW, Smith SL (2016) Wide field-ofview, multi-region, two-photon imaging of neuronal activity in the mammalian brain. Nat Biotechnol 34:857-862.

Stringer C, Pachitariu M, Steinmetz N, Carandini M, Harris KD (2019) High-dimensional geometry of population responses in visual cortex. Nature 571:361-365.

Tang J, Ardila Jimenez SC, Chakraborty S, Schultz SR (2016) Visual receptive field properties of neurons in the mouse LGN. PLoS One 11:e0146017.

Timme NM, Lapish C (2018) A tutorial for information theory in neuroscience. eNeuro 5:ENEURO.0052-18.2018.

Treves A, Panzeri S (1995) The upward bias in measures of information derived from limited data samples. Neural Comput 7:399-407.

Vogelstein JT, Packer AM, Machado TA, Sippy T, Babadi B, Yuste R, Paninski L (2010) Fast nonnegative deconvolution for spike train inference from population calcium imaging. J Neurophysiol 104:3691-3704.

Wachowiak M, Shipley MT (2006) Coding and synaptic processing of sensory information in the glomerular layer of the olfactory bulb. Semin Cell Dev Biol 17:411-423.

Wilent WB, Nitz DA (2007) Discrete place fields of hippocampal formation interneurons. J Neurophysiol 97:4152-4161. 
Yaksi E, Friedrich RW (2006) Reconstruction of firing rate changes across neuronal populations by temporally deconvolved $\mathrm{Ca} 2+$ imaging. Nat Methods 3:377-383.

Yartsev MM, Ulanovsky N (2013) Representation of three-dimensional space in the hippocampus of flying bats. Science 340:367-372.

Zhang K, Ginzburg I, McNaughton BL, Sejnowski TJ (1998) Interpreting neuronal population activity by reconstruction: unified framework with application to hippocampal place cells. J Neurophysiol 79:1017-1044.
Zhou JL, Shatskikh TN, Liu X, Holmes GL (2007) Impaired single cell firing and long-term potentiation parallels memory impairment following recurrent seizures. Eur J Neurosci 25:3667-3677.

Zinyuk L, Kubik S, Kaminsky Y, Fenton AA, Bures J (2000) Understanding hippocampal activity by using purposeful behavior: place navigation induces place cell discharge in both task-relevant and task-irrelevant spatial reference frames. Proc Natl Acad Sci USA 97:3771-3776.

Ziv Y, Burns LD, Cocker ED, Hamel EO, Ghosh KK, Kitch LJ, El Gamal A, Schnitzer MJ (2013) Long-term dynamics of CA1 hippocampal place codes. Nat Neurosci 16:264-266. 